\title{
HIV and SARS-CoV-2 Co-Infection: What are the Risks?
}

\author{
Nicola Squillace (1D) \\ Elena Ricci $\mathbb{D}^{2}$ \\ Elisa Colella (iD) \\ Paolo Bonfanti (1D) \\ 'Infectious Diseases Unit ASST-Monza, \\ San Gerardo Hospital-University of \\ Milano-Bicocca, Monza, Italy; \\ ${ }^{2}$ Fondazione ASIA Onlus, Milan, Italy
}

\begin{abstract}
The dramatic increase of the global pandemic of SARS-CoV-2 infection represents a critical issue that needs to be investigated to evaluate the associated risk factors for acquisition and worse outcome. The interplay between immune activation and immune depression during SARS-CoV-2 infection is an intriguing topic that still needs to be clarified. The role of HIV in SARS-CoV-2 infection is not well defined. Chronic inflammation linked to HIV infection could be a driver for a worse prognosis in people living with HIV (PLWH). We explored the role of HIV as a risk factor for SARS-CoV-2 infection and severity and which factors contributed to a worse prognosis when HIV infection was present. PubMed/ MEDLINE was searched for "COVID-19" or "SARS-CoV2" and "HIV" or "AIDS" and ("hospitalization" or "intensive care" or "mechanical ventilation" or "death" OR "mortality"), both in MeSH and as free text in all fields. Our review focused on 21 studies that enrolled at least 40 PLWH. In most studies, HIV infection did not represent a risk factor for SARS-CoV-2 infection. On the contrary, the risk of severe COVID-19 and hospitalization was higher in PLWH. Low CD4 cell count consistently emerged as a risk factor for severe COVID-19. Comorbidities, either in people with or without HIV diagnosis, played a key role, especially because of their early development in PLWH.
\end{abstract}

Keywords: HIV, SARS, CoV-2, COVID-19

\section{Introduction}

As of early June 2021, more than 170 million cases of SARS-CoV-2 disease (COVID-19) were diagnosed, with over 3.7 million deaths worldwide (https:// covid19.who.int/).

High mortality rates are associated with progressive respiratory failure and acute kidney injury ${ }^{1}$ and are linked to systemic microvascular thrombosis and generalized coagulopathy, both related to high inflammatory response and continuing complement activation.

Previous health conditions have been consistently identified as risk factors for severe COVID-19 outcomes, mainly obesity, hypertension, diabetes, and chronic pulmonary disease. Due to the accelerated aging linked to persistent inflammation, cardiovascular comorbidities are more frequent among people living with HIV (PLWH) than in the general population. ${ }^{2}$ Given this disproportionate burden, a larger number of severe cases and deaths are expected among PLWH.

HIV-1 and SARS-CoV-2 infection share CD4+T cells (CD4) loss in association with disease outcome and immunodeficiency. In both diseases, immune activation, direct attacks on CD4, and redistribution of CD4 contribute, in quite different proportions, to CD4 lymphopenia. Like for HIV, lymphopenia and marked CD4
Infectious Diseases Unit ASST-Monza, San Gerardo Hospital-University of MilanoBicocca, via G.B. Pergolesi 33, Monza, 20900, Italy

Email n.squillace@asst-monza.it; nicolasquillace74@gmail.com 
count reduction in COVID-19 patients have been linked with poor clinical outcomes. However, when HIV and COVID-19 meet, no additional decrease of CD4 count has been observed. ${ }^{3}$ PLWH could even experience protection from the most serious sequelae of COVID-19, because of their history of immune response or the hypothesized activity of Anti-Retroviral Treatment (ART) against SARS-CoV-2., ${ }^{4,5}$

The interplay between SARS-CoV-2 and HIV is affected by several conflicting aspects. The residual chronic inflammation, present even in suppressed PLWH, and the immune deficiency due to HIV may exert opposite effects. The age might also affect the outcomes in coinfected COVID-19HIV patients, who may be younger and monitored for comorbidities at an earlier age than their HIV-negative counterparts. The conflicting evidence about their interaction may be due to the relative weight of these factors. ${ }^{6}$

However, evidence regarding the clinical outcome of SARS-CoV-2 in PLWH is still inconsistent.

For example, in a single-center cohort study, PLWH diagnosed with COVID-19 was not different from the COVID-19 patients without known HIV diagnosis. ${ }^{7}$ Clinical presentation, severity of the disease, and mortality did not depend on HIVrelated or ART-related factors. The standardized incidence rate of COVID-19 was lower in PLWH than in the Barcelona general population, although no comparison of mortality rate was performed between the two groups. Similarly, a propensity matched analysis revealed no difference between HIV+ and HIV- subjects, suggesting that higher mortality was likely driven by a higher number of comorbidities. ${ }^{8}$

On the contrary, a comparison between HIV-negative and positive patients, admitted in 207 hospitals across the United Kingdom, showed higher day-28 mortality in PLWH, after considering potential risk factors such as age, sex, comorbidities and need for oxygen at presentation. In particular, in people aged less than 60 years the adjusted hazard ratio (aHR) was 2.87 (95\% confidence interval, CI, 1.70-4.86), increased risk due to HIV status. ${ }^{9}$

The objective of this review was to explore the role of HIV infection as risk factor for COVID-19 diagnosis, hospitalization, and death, and to investigate the risk factors for COVID-19 severity in PLWH.

\section{Methods}

\section{Database Search}

PubMed/MEDLINE was searched for "COVID-19" or "SARS-CoV-2" and "HIV" or "AIDS" and ("hospitalization" or "intensive care" or "mechanical ventilation" or "death" OR "mortality"), both in MeSH and as free text in all fields (limits: Human, English). The search was limited to articles published in English until 15 May 2021, retrieving 552 results in PubMed/MEDLINE.

We excluded papers that reported protocols, no information about HIV status, laboratory studies, case reports, and editorials.

Among 47 studies reporting on COVID-19 outcomes in PLWH, we evaluated 21 studies with at least $40 \mathrm{PLWH}$ enrolled and analyzed HIV infection as risk factor for the different considered outcomes.

Bibliography from the retrieved articles was manually searched to identify further relevant literature about the relationship between SARS-CoV-2 and HIV.

\section{HIV Infection and Its Impact on SARS-CoV-2 \\ Infection}

The main characteristics of selected studies are reported in Tables 1 and 2 .

\section{HIV Infection and COVID-19 Diagnosis}

Most cohort studies did not show a higher incidence of COVID-19 in PLWH than in the general population (Table 1). ${ }^{7,10-13}$

In a large cohort study ${ }^{10}$ that involved 2988 COVID-19 positive PLWH, similar diagnosis rates for COVID-19 were observed in PLWH and in people not living with known HIV diagnosis (PNLWH) after standardization (standardized rate ratio, sRR, 0.94, 95\% CI, 0.91-0.97). In an adjusted model, PLWH of older age, not white nonHispanic race/ethnicity, and living in the regions of metropolitan New York City (NYC) were significantly more likely to receive a diagnosis of COVID-19. No significant differences were observed between the main HIV transmission groups. In adjusted analysis, stage $3 \mathrm{HIV}$ infection was related with an increased rate of COVID-19 diagnosis (vs stage 1: adjusted RR 1.22, 95\% CI 1.07-1.38).

Cabello et $\mathrm{al}^{11}$ described an infection rate between 0.86 (confirmed cases) and 1.68 (confirmed and suspected cases), similar to Madrid general population.

An observational prospective study $^{14}$ conducted on 2873 PLWH observed 51 COVID-19 infections (incidence $1.8 \%, 95 \%$ CI, 1.3-2.3). Although the rate of suspected and confirmed cases in the general population was higher (4.02\%, 95\% CI 4.01-4.03\%), the rate of confirmed diagnosis was similar among PLWH and the general 


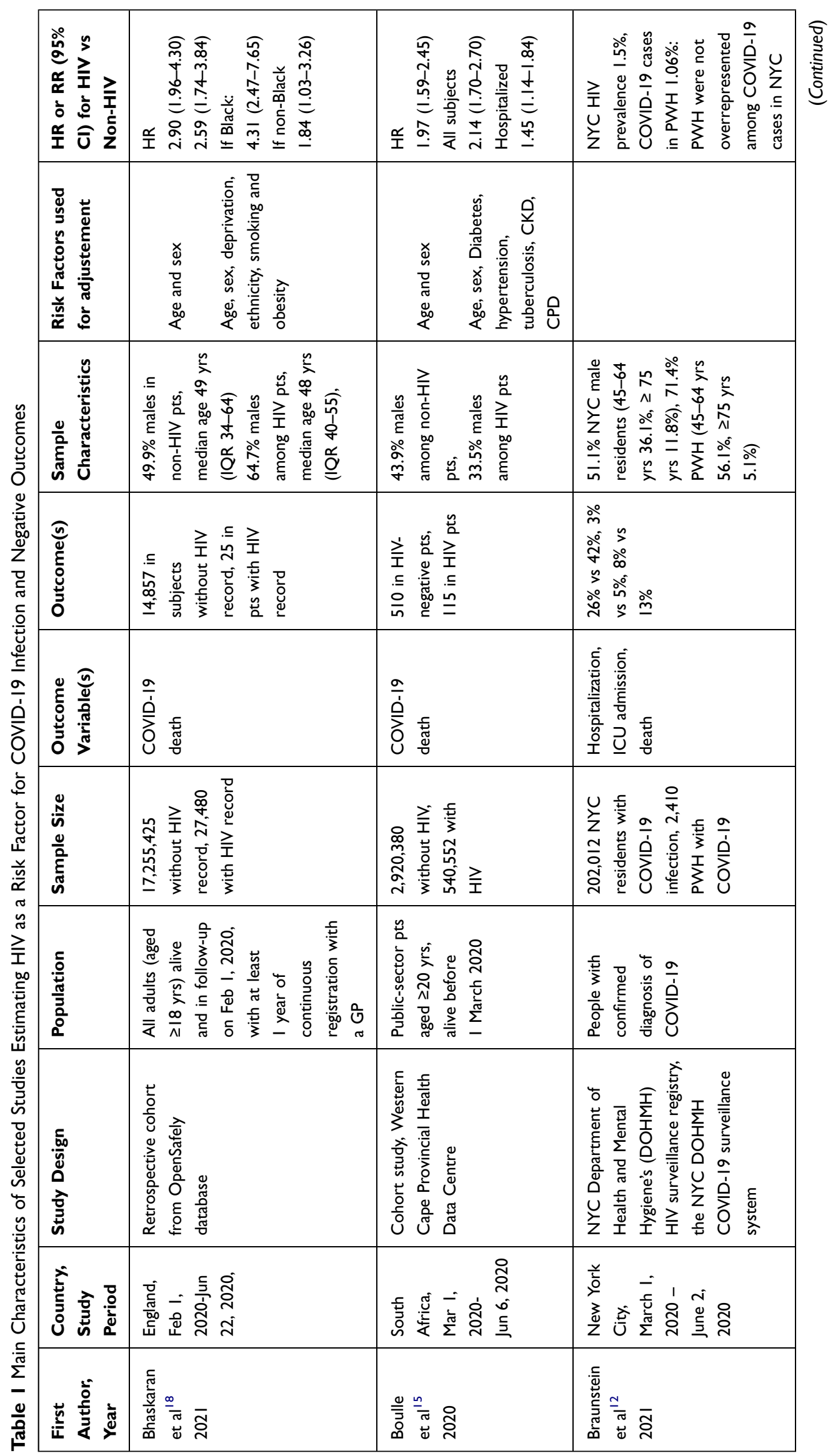




\begin{tabular}{|c|c|c|c|}
\hline 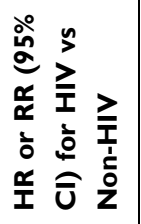 & 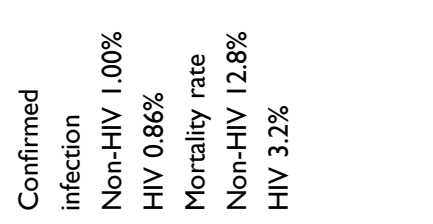 & 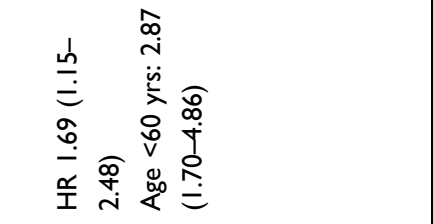 & 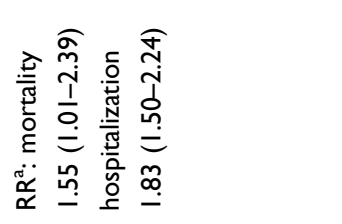 \\
\hline 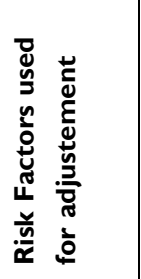 & & 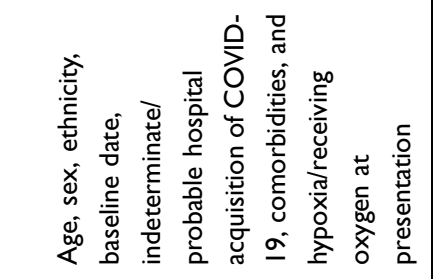 & \\
\hline 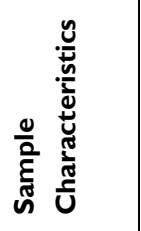 & & 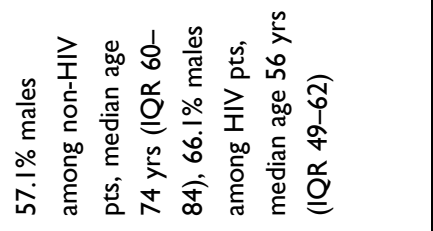 & 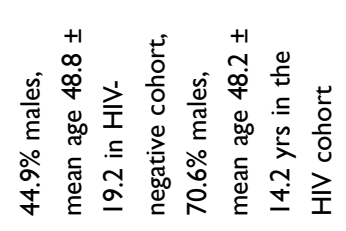 \\
\hline 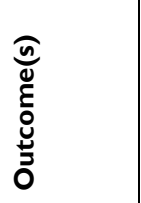 & 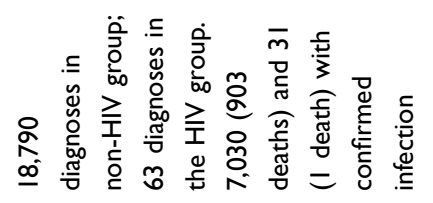 & 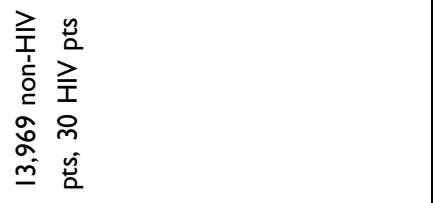 & 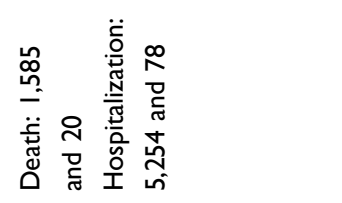 \\
\hline 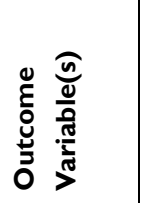 & 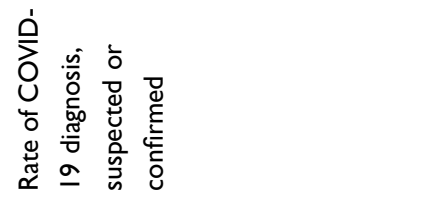 & 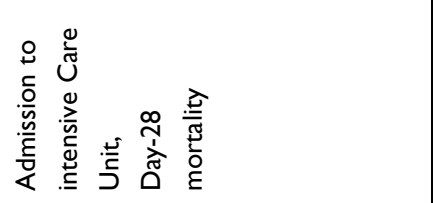 & 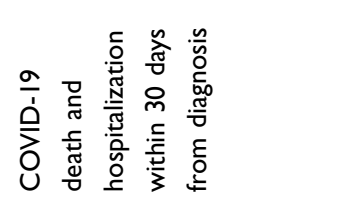 \\
\hline 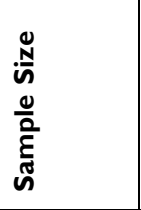 & 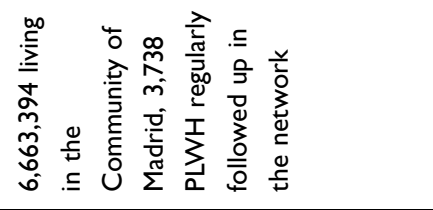 & 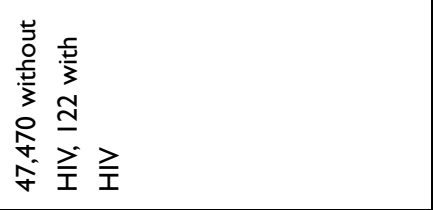 & 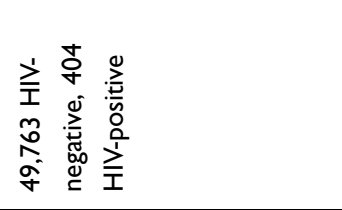 \\
\hline 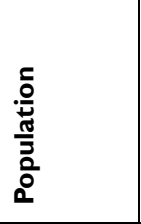 & 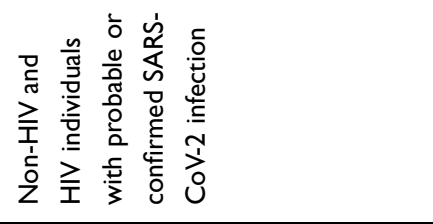 & 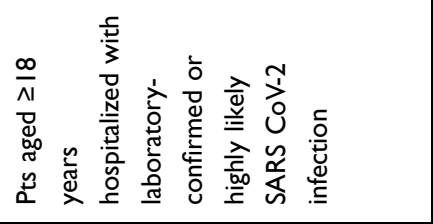 & 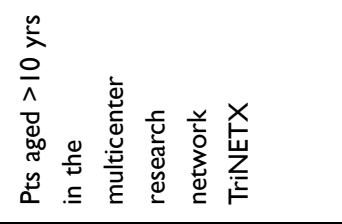 \\
\hline 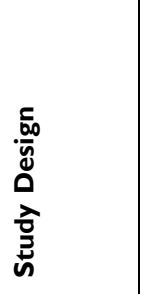 & 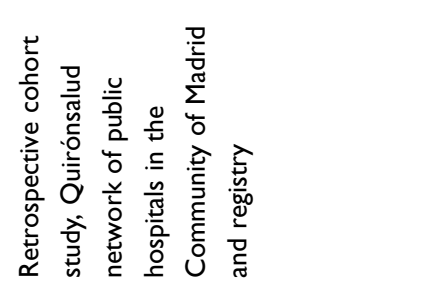 & 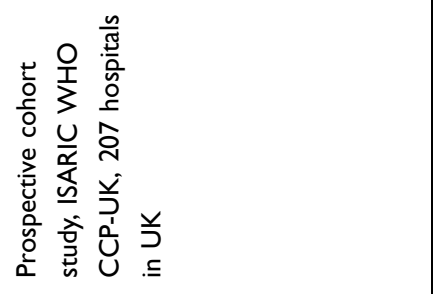 & 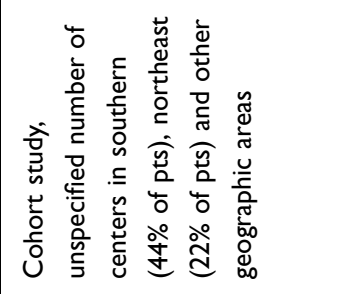 \\
\hline 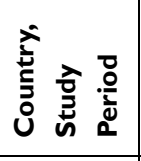 & 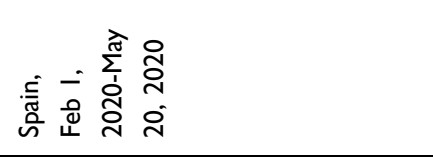 & 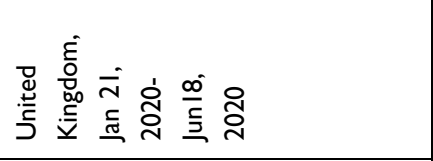 & 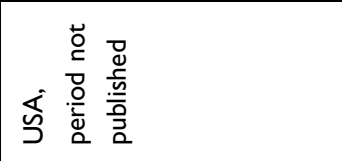 \\
\hline 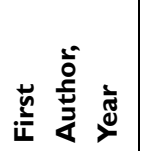 & 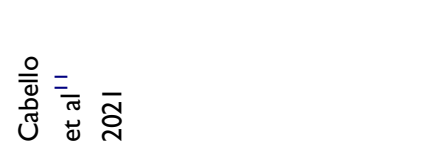 & 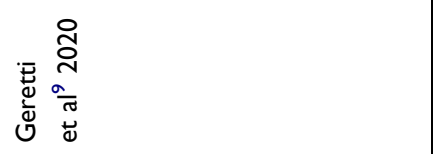 & 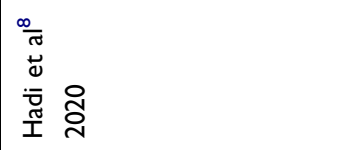 \\
\hline
\end{tabular}




\begin{tabular}{|c|c|c|c|}
\hline 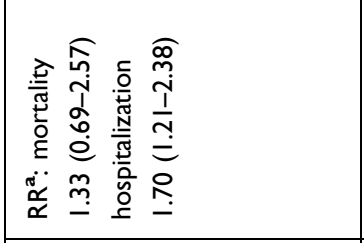 & 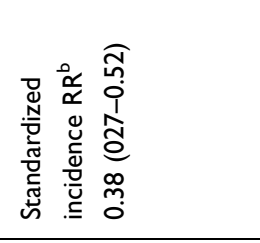 & 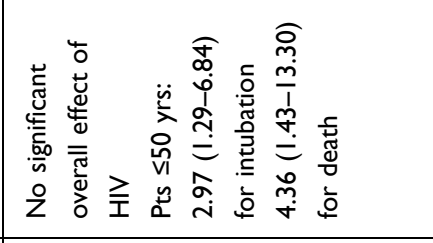 & 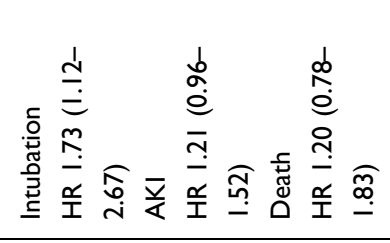 \\
\hline 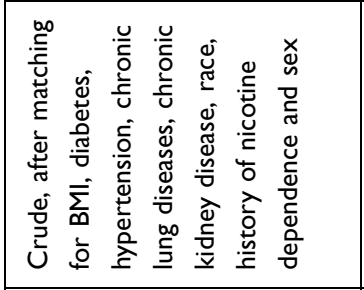 & 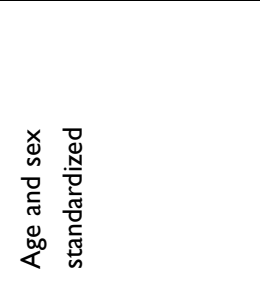 & & 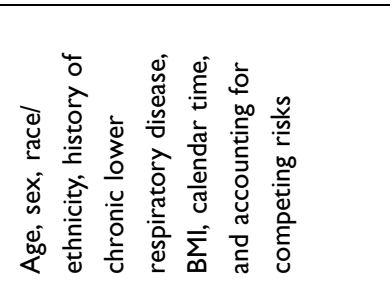 \\
\hline 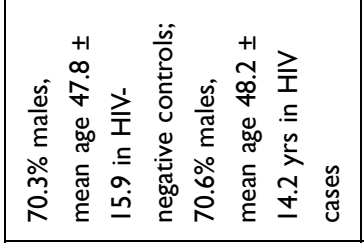 & & 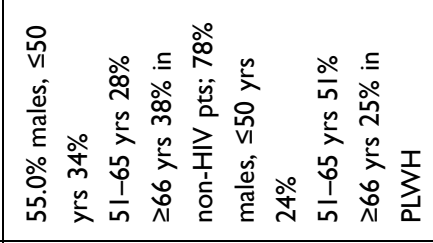 & 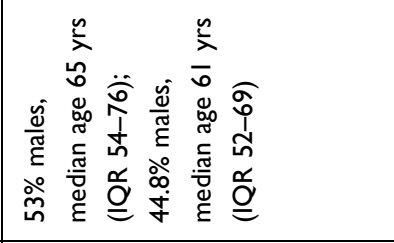 \\
\hline 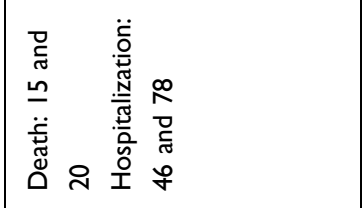 & 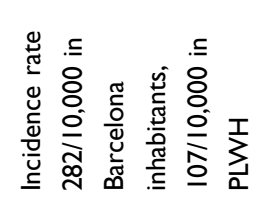 & 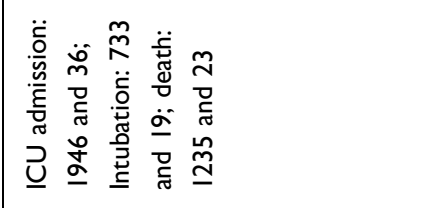 & 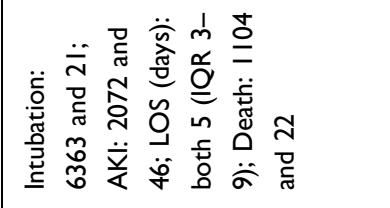 \\
\hline 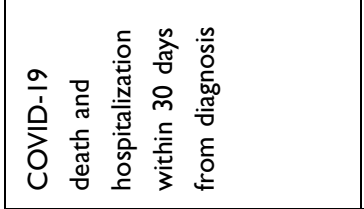 & 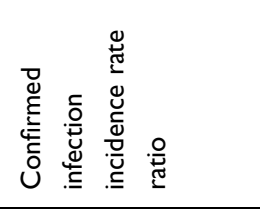 & 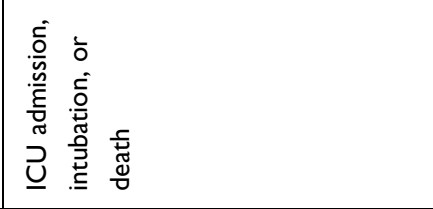 & 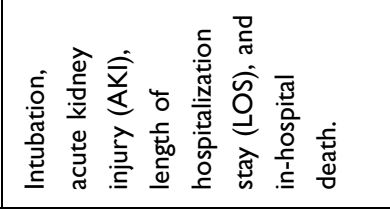 \\
\hline 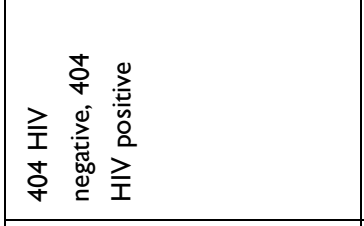 & 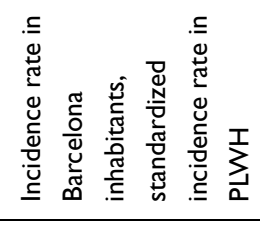 & 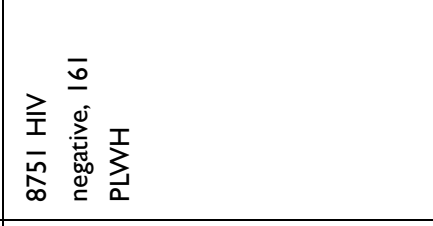 & 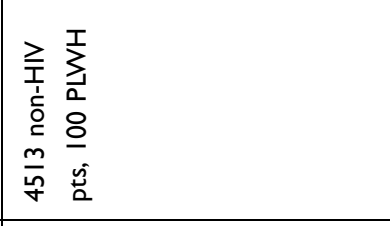 \\
\hline 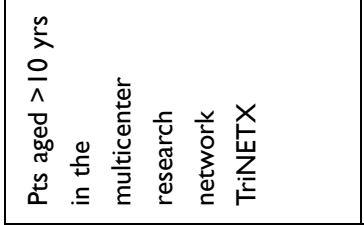 & 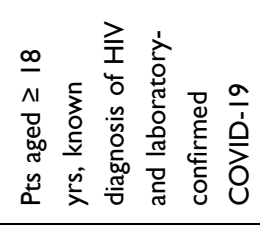 & 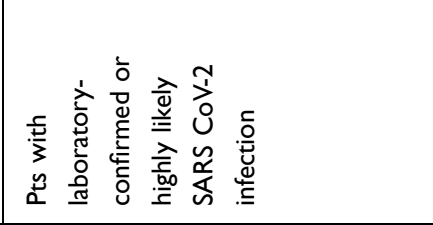 & 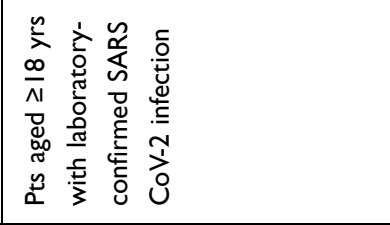 \\
\hline 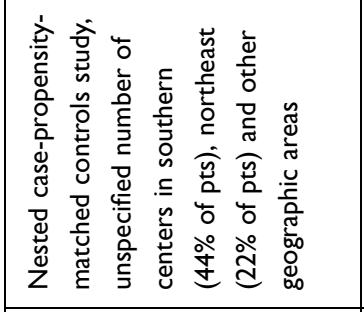 & 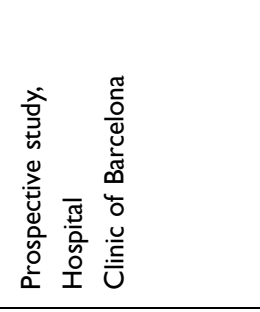 & 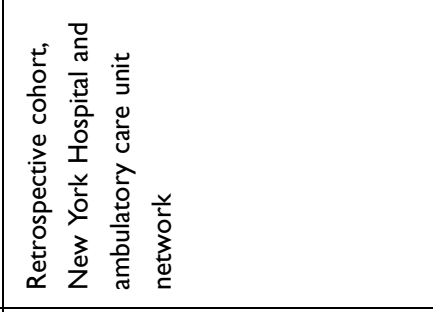 & 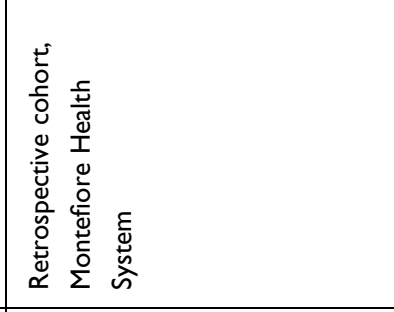 \\
\hline 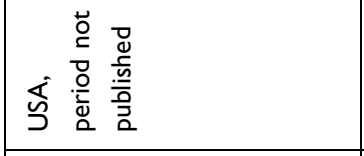 & 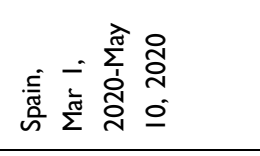 & 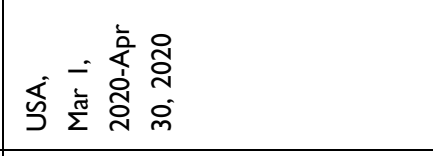 & 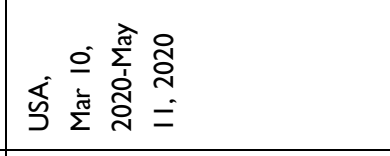 \\
\hline 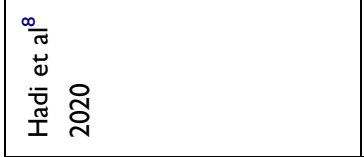 & 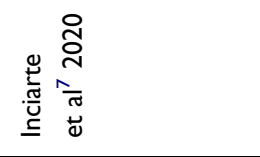 & 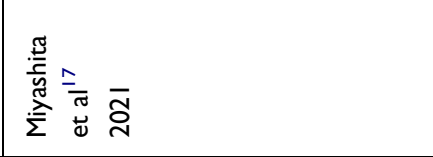 & 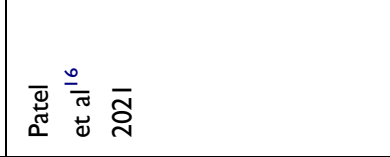 \\
\hline
\end{tabular}




\begin{tabular}{|c|c|c|c|}
\hline 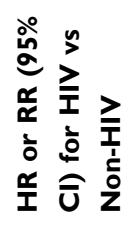 & 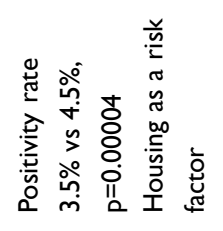 & 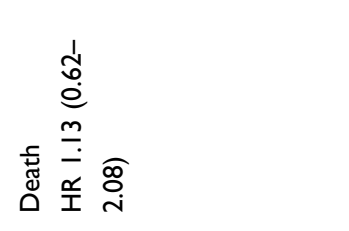 & 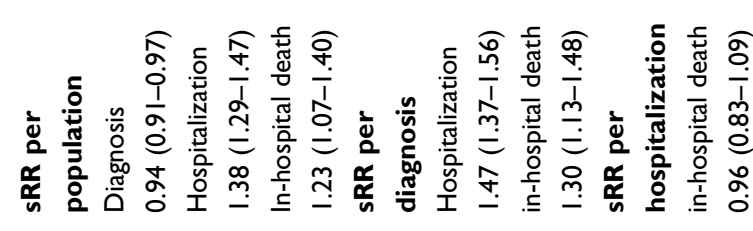 \\
\hline 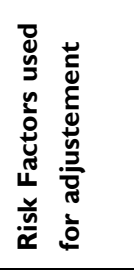 & & 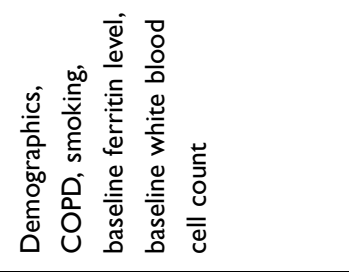 & 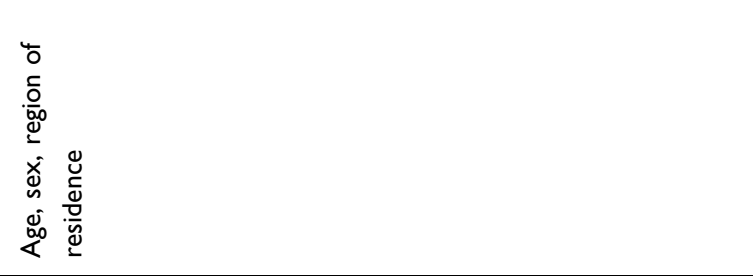 \\
\hline 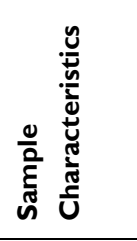 & 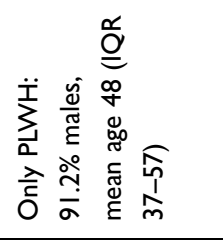 & 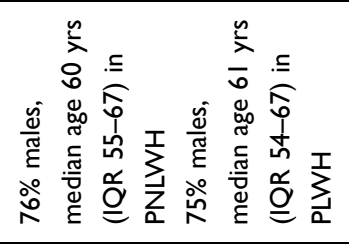 & 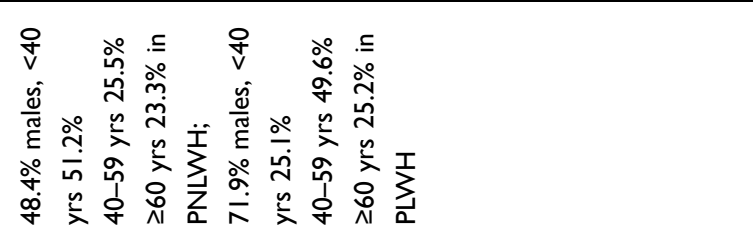 \\
\hline 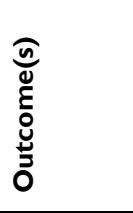 & 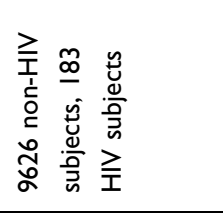 & 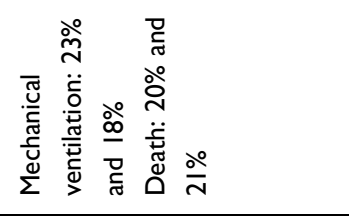 & 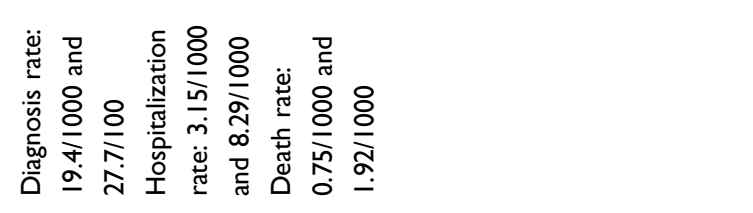 \\
\hline 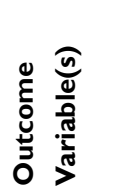 & 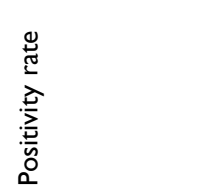 & 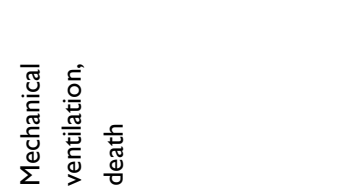 & 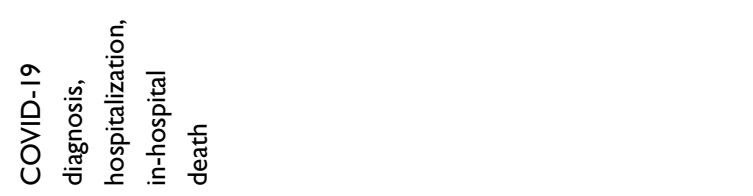 \\
\hline $\begin{array}{l}\frac{N}{N} \\
\frac{0}{0} \\
\frac{0}{0} \\
\text { ज๊ }\end{array}$ & 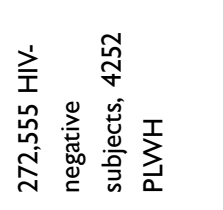 & 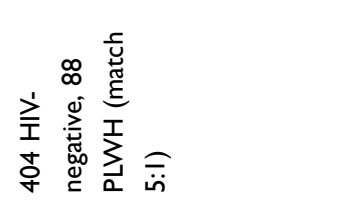 & 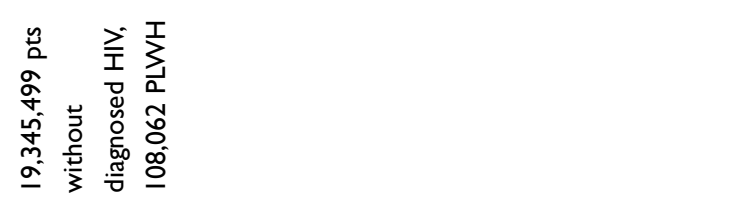 \\
\hline 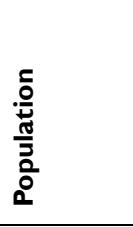 & 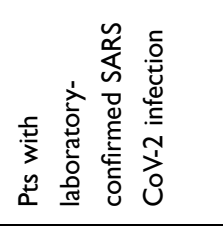 & 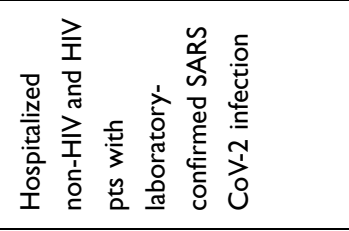 & 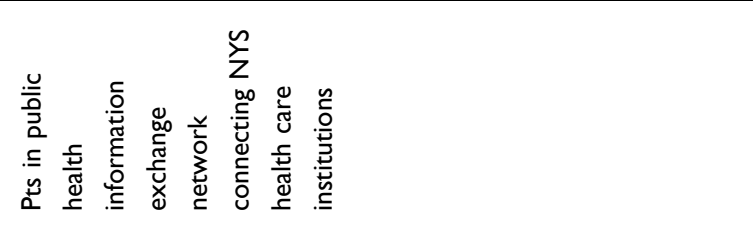 \\
\hline 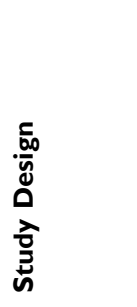 & 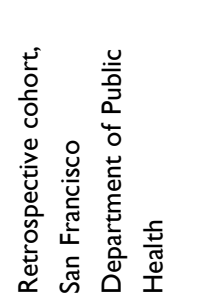 & 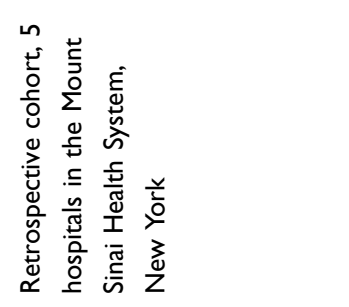 & 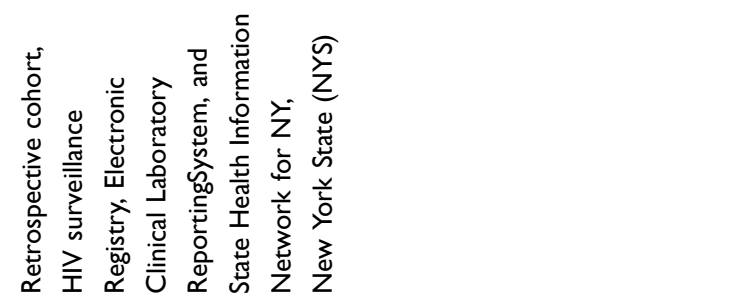 \\
\hline 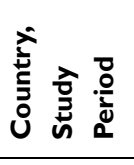 & 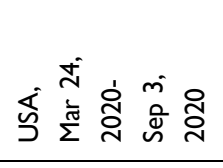 & 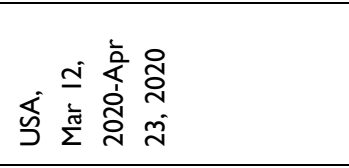 & 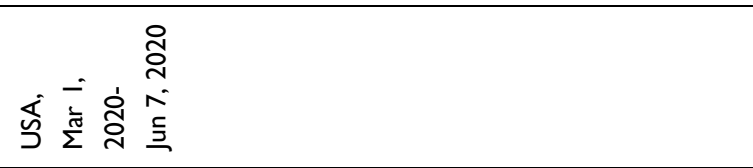 \\
\hline 䓂竞㐫 & 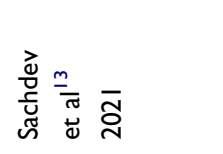 & 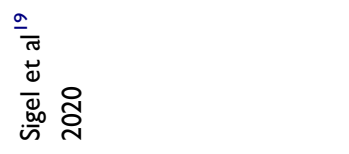 & 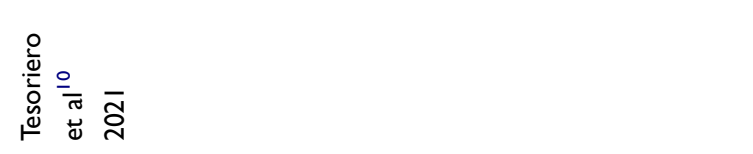 \\
\hline
\end{tabular}




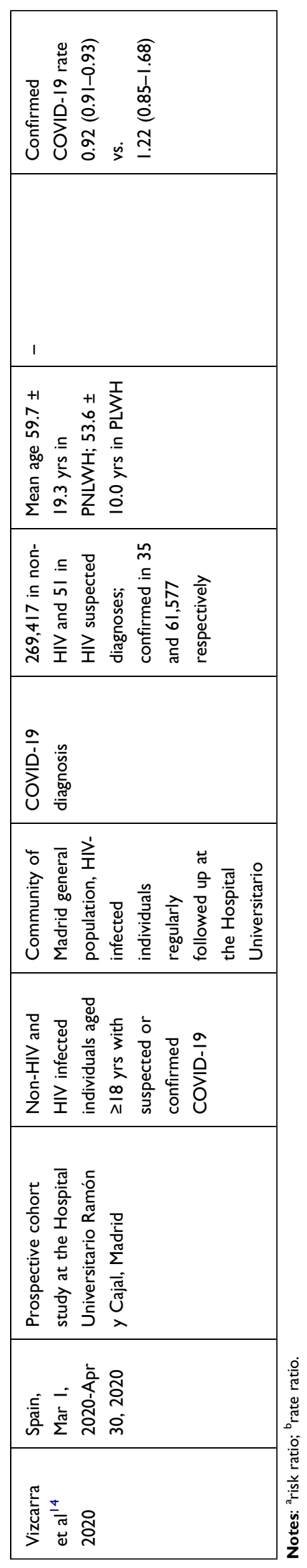

population $(0.92 \%, 95 \%$ CI $0.91-0.93 \%$, and $1.22 \%, 95 \%$ CI $0.85-1.68 \%$ respectively).

On the same line, Braunstein et $\mathrm{al}^{12}$ observed that COVID-19 did not disproportionately affect the PLWH in NYC, since the diagnosed HIV with COVID-19 represented $1.06 \%$ of all confirmed COVID-19 cases, and the overall prevalence of HIV in the NYC population was $1.5 \%$ in 2018.

On the contrary, in the analysis from Inciarte et al, ${ }^{7}$ the incidence of confirmed COVID-19 cases in a cohort of 5683 PLWH was $62 \%$ lower than in the Barcelona population.

On the other hand, Sachdev et $\mathrm{al}^{13}$ found a significantly higher diagnosis rate in 4252 PLWH compared to PNLWH (4.5\% vs $3.5 \%, \mathrm{p}=0.00004)$.

Finally, we considered the prevalence of comorbidities in PLWH with or without COVID-19. Boulle et $\mathrm{al}^{15}$ described a slightly lower prevalence of diabetes ( $4 \%$ vs $11 \%)$ and hypertension (12\% vs $19 \%)$ in PLWH without COVID-19 vs PLWH with COVID-19. Vizcarra et $\mathrm{al}^{14}$ confirmed the lower prevalence of diabetes and hypertension in PLWH without COVID-19, also finding a lower percentage of patients with chronic kidney disease and liver disease in this subset of patients.

\section{HIV Infection and Hospitalization for COVID-19}

Many studies demonstrated a higher rate of hospitalization in PLWH than in PNLWH (Table 1). ${ }^{8,10,12,14}$

Tesoriero et al $^{10}$ observed population-level rates of COVID-19 hospitalization significantly higher among PLWH (8.28 per 1000) than among PNLWH (3.15 per 1000; sRR 1.38, 95\% CI, 1.29-1.47). In the adjusted analysis, older age and region were associated with hospitalization. This study observed an increasing hospitalization risk across stage 1, 2 and 3 of HIV infection: more in detail, a per-person hospitalization standardized rate analysis was conducted by stage of HIV disease. Compared to people living without HIV infection, hospitalization risk was progressively higher by HIV stage 1 infection: sRR was 1.19 (95\% CI 1.08-1.30) for stage 1, 1.60 (95\% CI 1.42-1.78) for stage 2 , and 2.66 (95\% CI 2.20-3.13) for stage 3 infection. Hadi et $\mathrm{al}^{8}$ with their large analysis conducted on more than fifty thousand COVID-19 patients (404 with preexisting HIV diagnosis) confirmed a significantly higher proportion of hospitalization in the HIV-positive group (19.3\% vs $11.4 \%$; risk ratio $1.70,95 \%$ CI 1.21-2.38), after propensity score matching for body mass index (BMI), diabetes, hypertension, chronic lung 


\begin{tabular}{|c|c|c|c|c|}
\hline 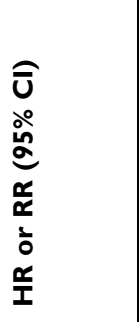 & 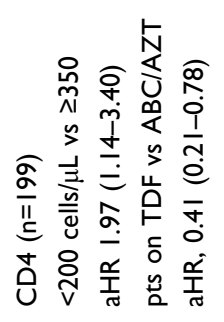 & 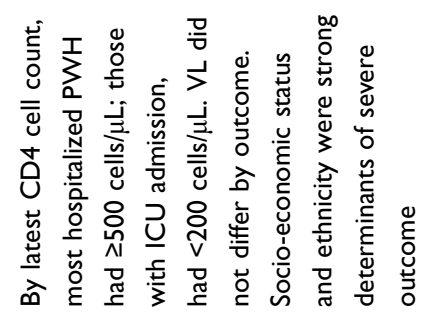 & 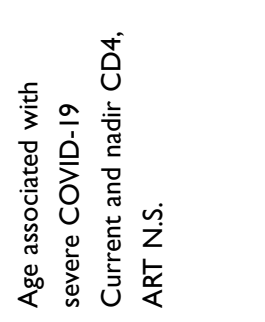 & 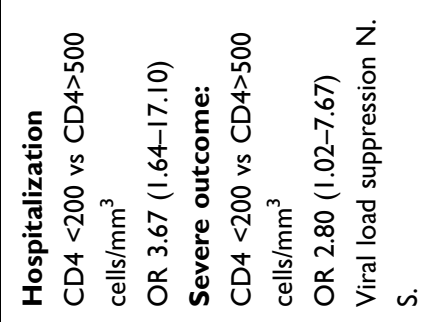 \\
\hline 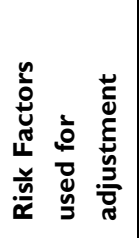 & 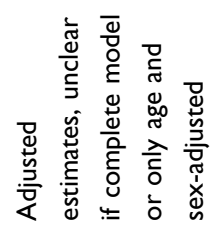 & 1 & 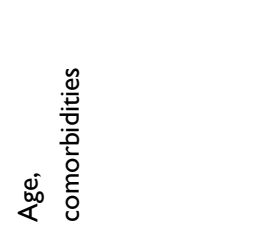 & 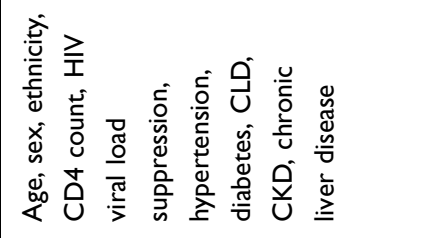 \\
\hline 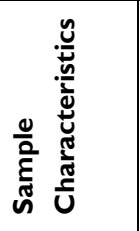 & 1 & 1 & 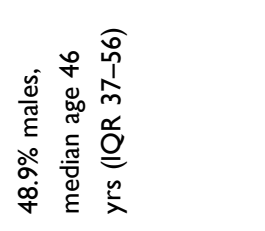 & 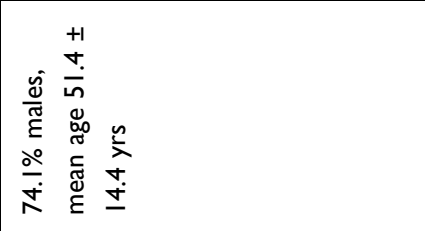 \\
\hline 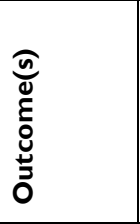 & 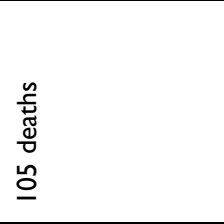 & 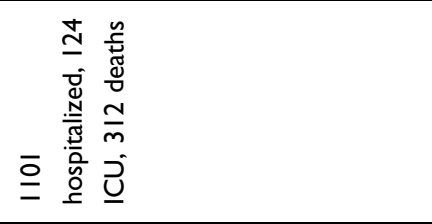 & 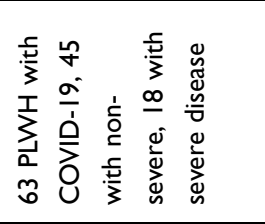 & 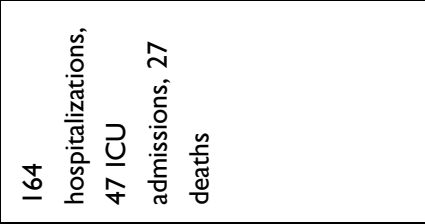 \\
\hline 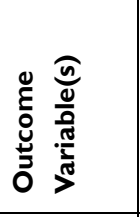 & 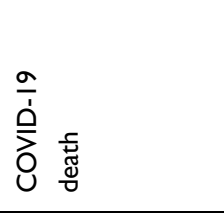 & 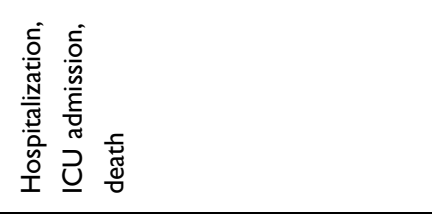 & 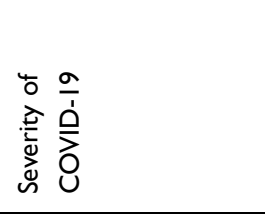 & 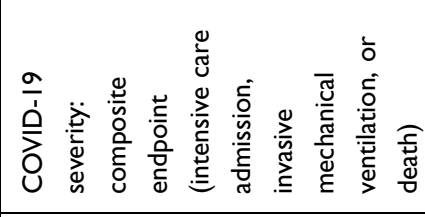 \\
\hline 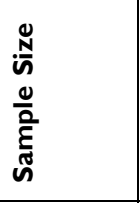 & 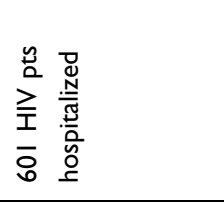 & 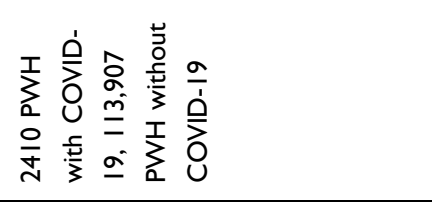 & 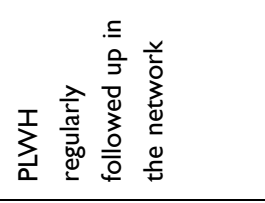 & 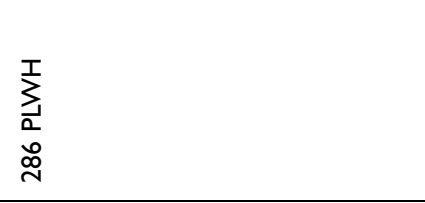 \\
\hline 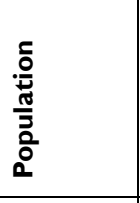 & 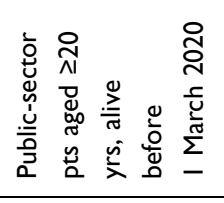 & 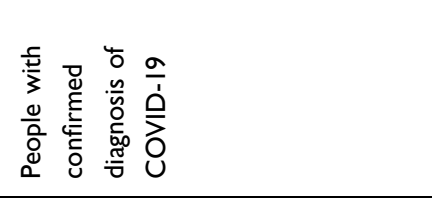 & 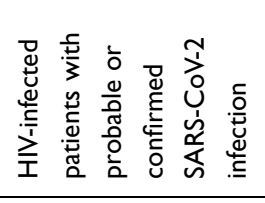 & 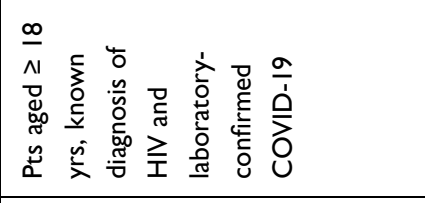 \\
\hline 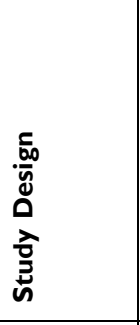 & 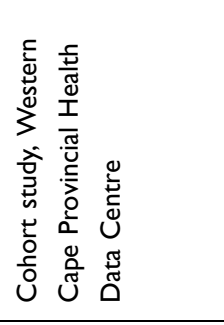 & 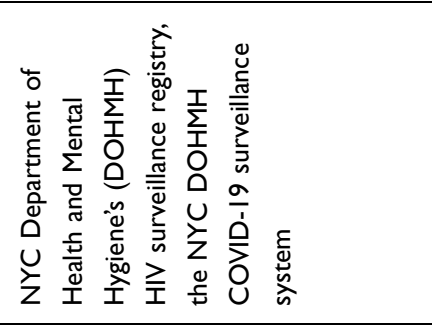 & 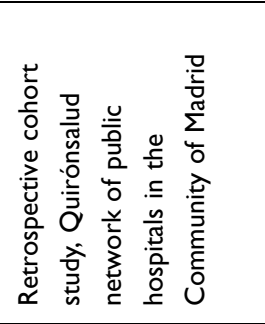 & 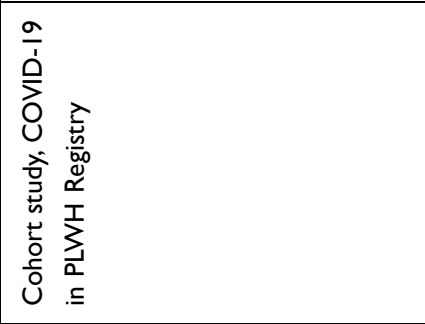 \\
\hline 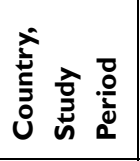 & 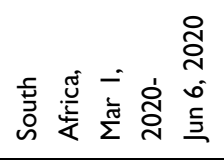 & 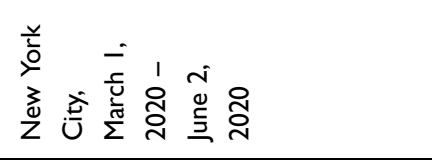 & 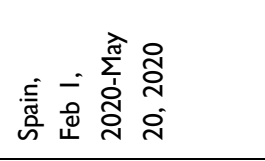 & 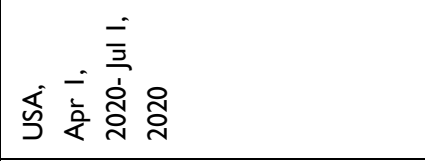 \\
\hline 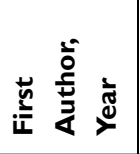 & $\frac{0}{\bar{J}} \frac{n}{\bar{J}}$ & 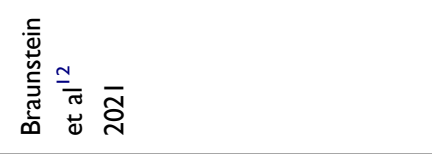 & 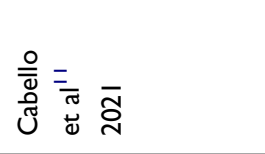 & 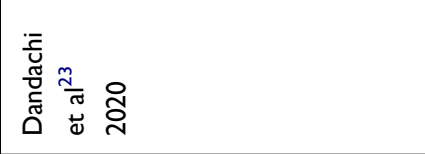 \\
\hline
\end{tabular}




\begin{tabular}{|c|c|c|c|}
\hline 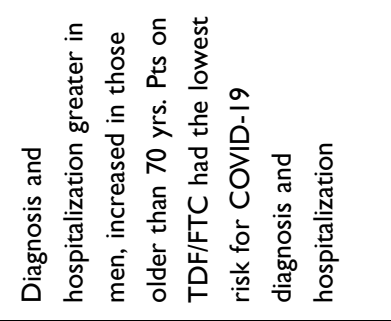 & 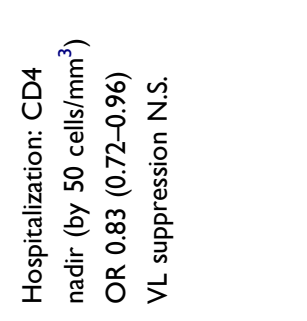 & 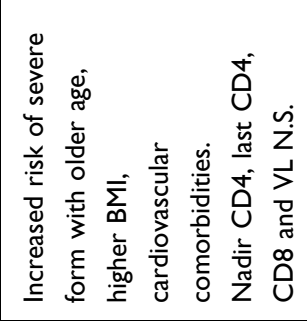 & 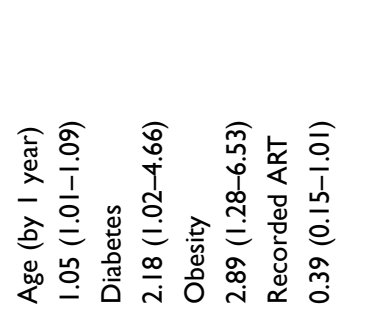 \\
\hline 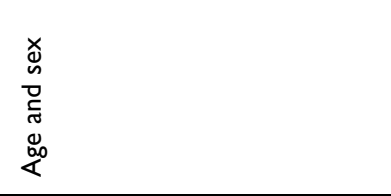 & 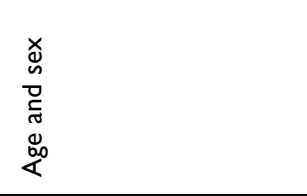 & 1 & 1 \\
\hline 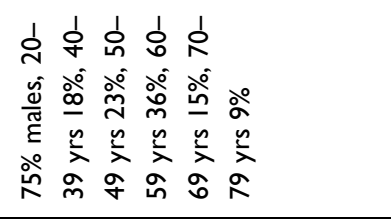 & 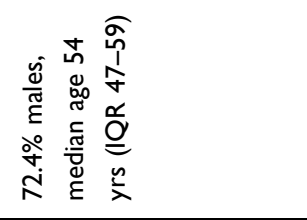 & 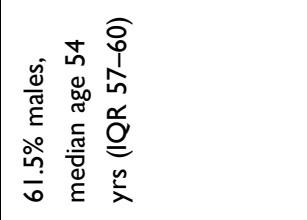 & 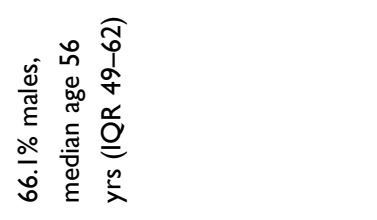 \\
\hline 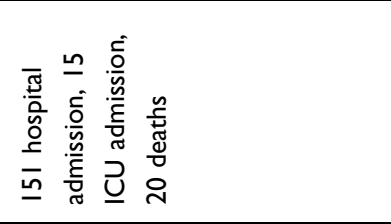 & 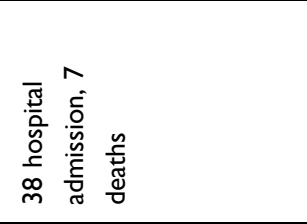 & 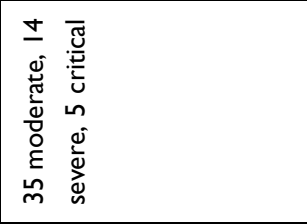 & 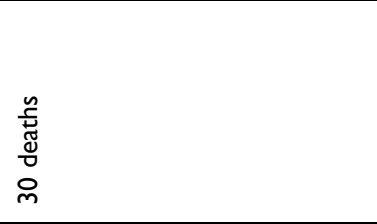 \\
\hline 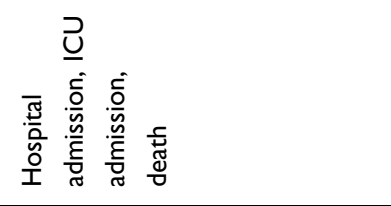 & 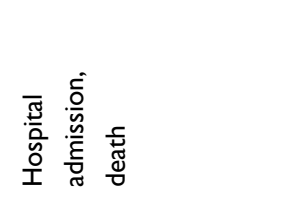 & 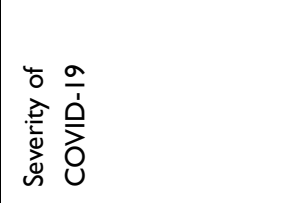 & 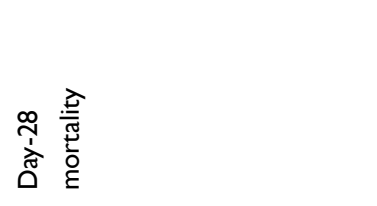 \\
\hline 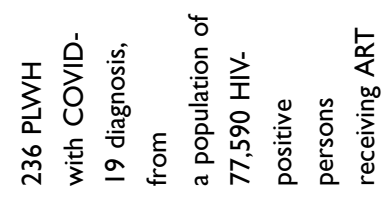 & & 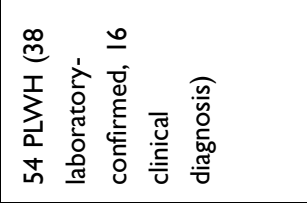 & 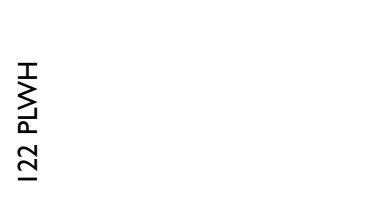 \\
\hline 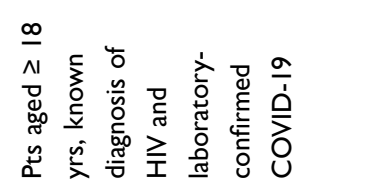 & 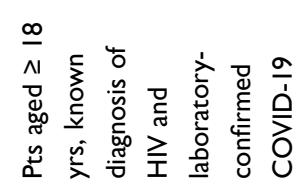 & 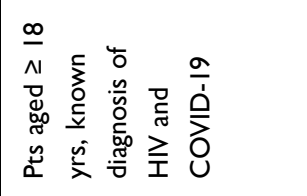 & 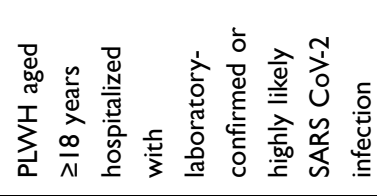 \\
\hline 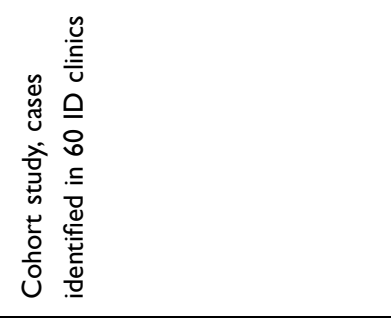 & 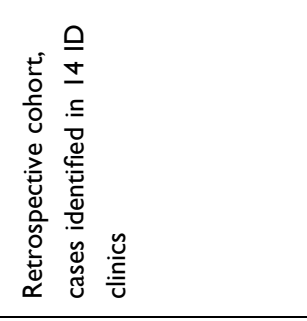 & 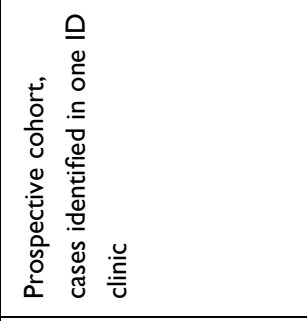 & 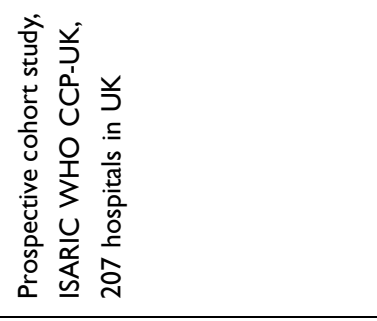 \\
\hline 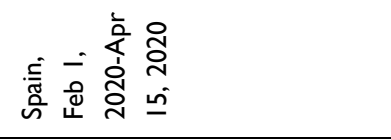 & 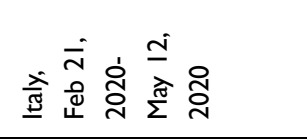 & 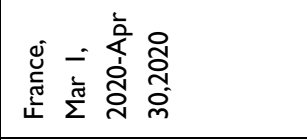 & 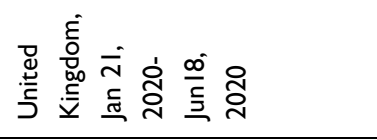 \\
\hline 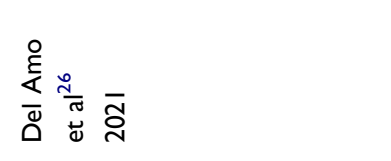 & 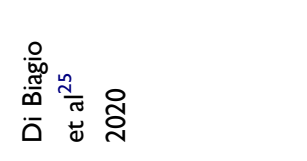 & 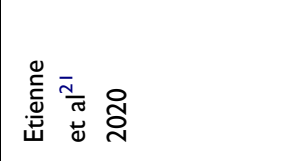 & 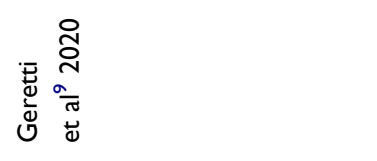 \\
\hline
\end{tabular}




\begin{tabular}{|c|c|c|c|c|}
\hline 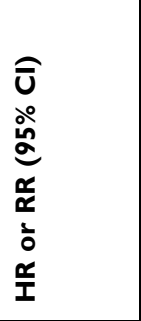 & 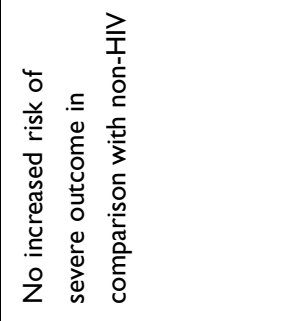 & 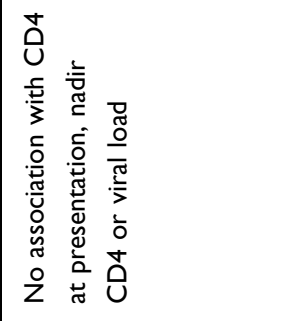 & 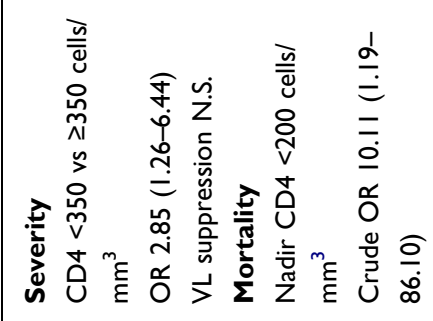 & 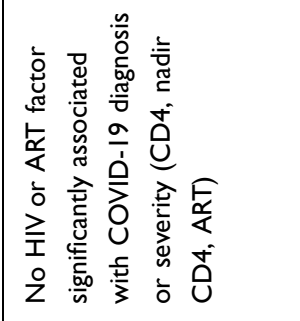 \\
\hline 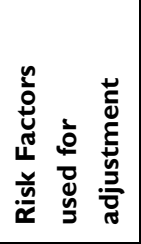 & 1 & . & 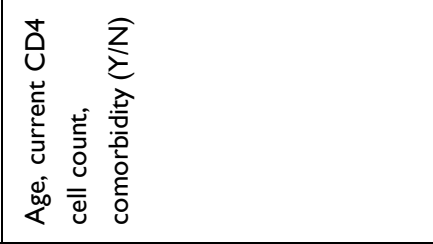 & \\
\hline 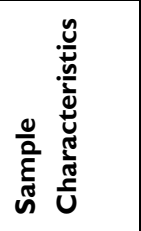 & 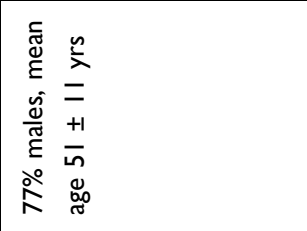 & 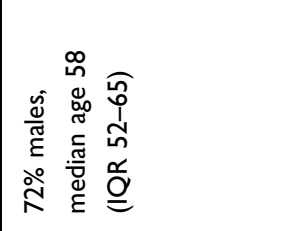 & 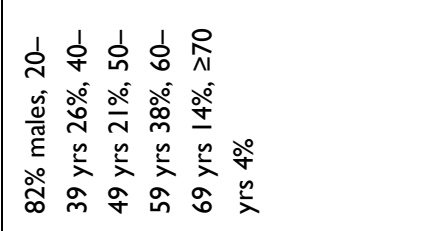 & 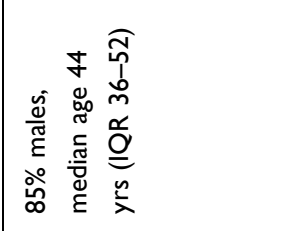 \\
\hline 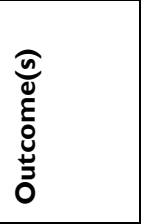 & 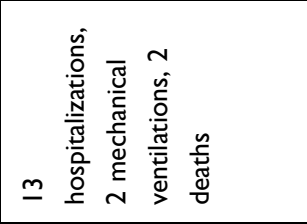 & 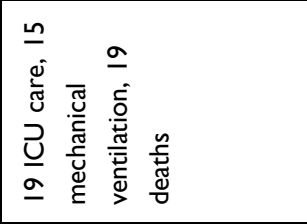 & 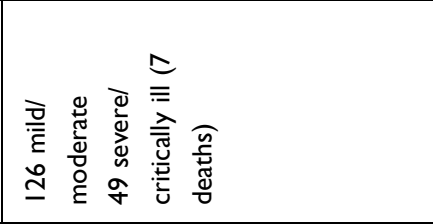 & 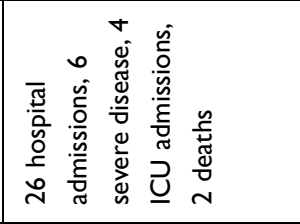 \\
\hline 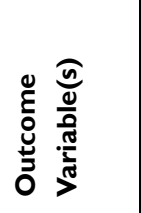 & 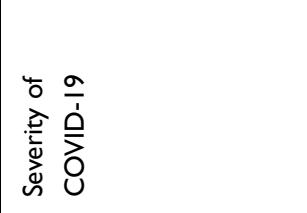 & 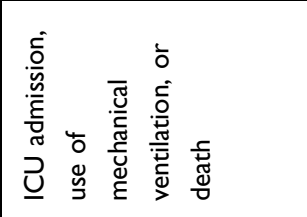 & 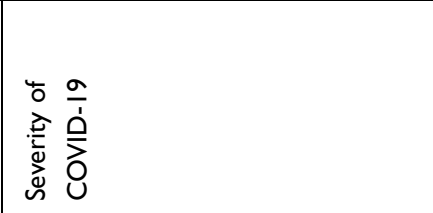 & 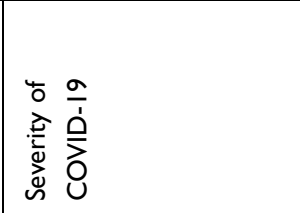 \\
\hline 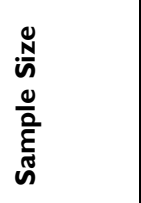 & 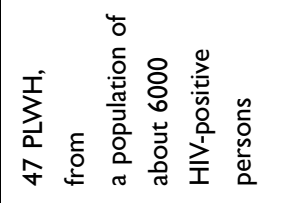 & 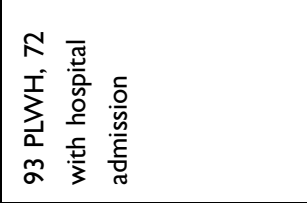 & $\begin{array}{l}\sum_{2}^{T} \\
\underline{L} \\
\underline{\Lambda}\end{array}$ & 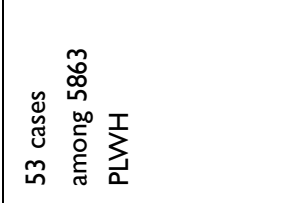 \\
\hline 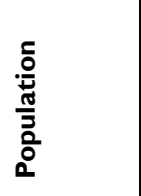 & 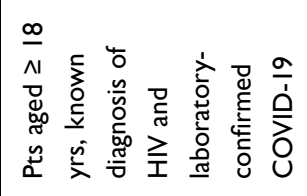 & 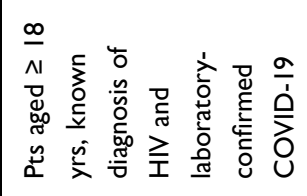 & 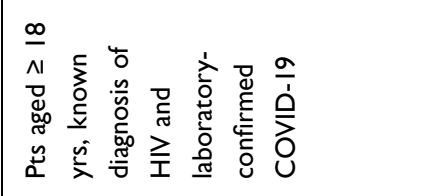 & 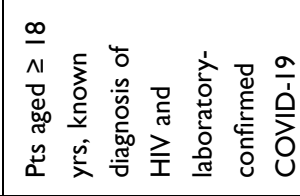 \\
\hline 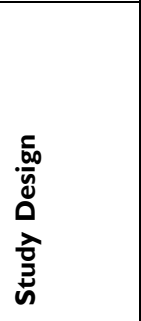 & 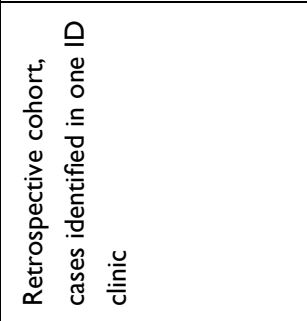 & 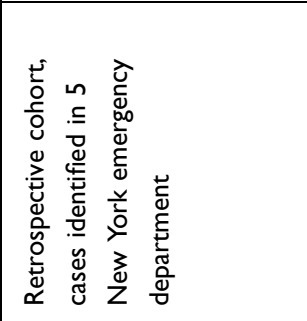 & 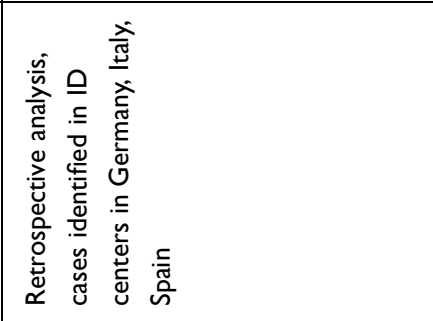 & 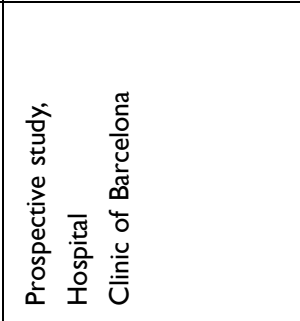 \\
\hline 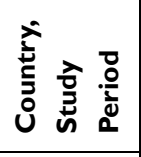 & 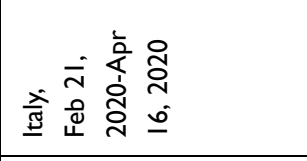 & 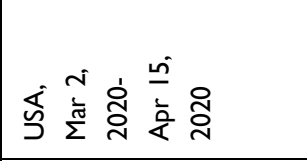 & 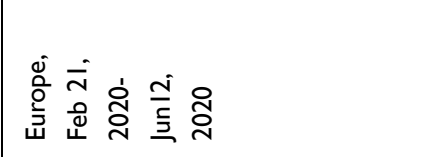 & 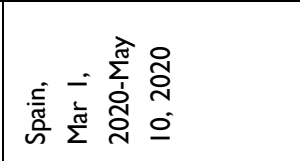 \\
\hline 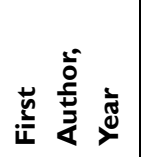 & 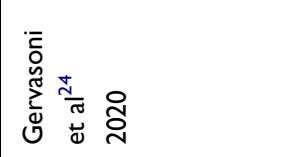 & $\begin{array}{l}\frac{1}{\pi} \\
\stackrel{4}{0} \\
\stackrel{2}{0} \\
\stackrel{0}{2}\end{array}$ & 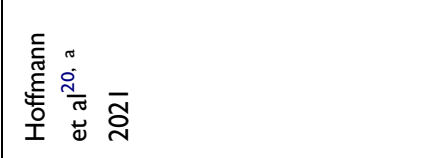 & 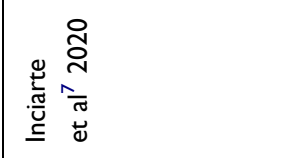 \\
\hline
\end{tabular}




\begin{tabular}{|c|c|c|}
\hline 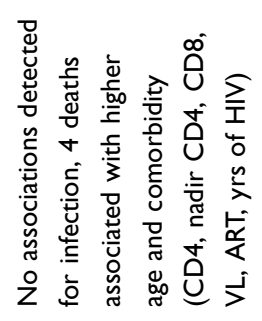 & 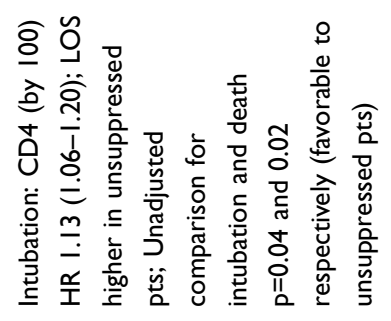 & 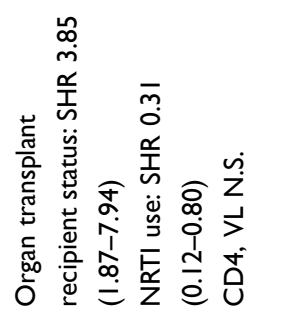 \\
\hline ' & 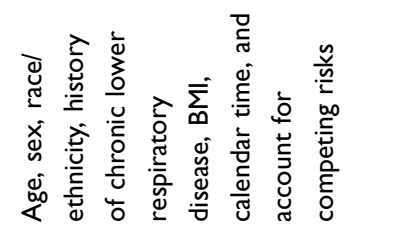 & 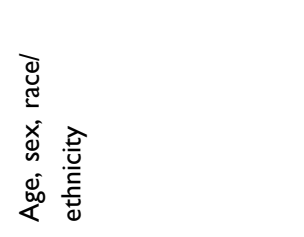 \\
\hline 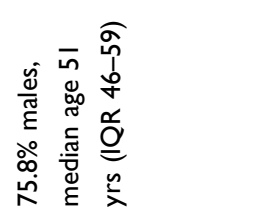 & 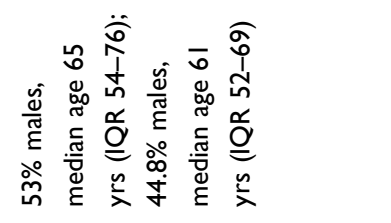 & 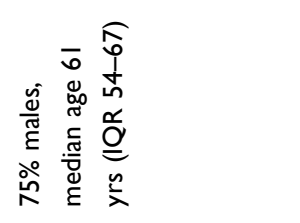 \\
\hline 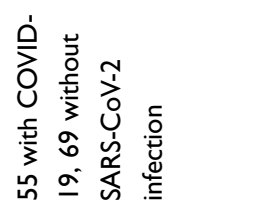 & 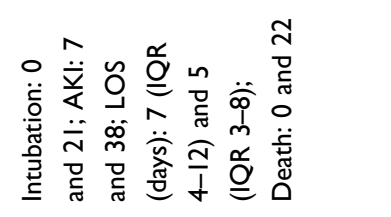 & 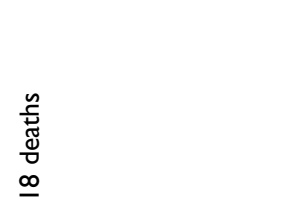 \\
\hline 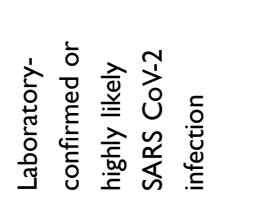 & 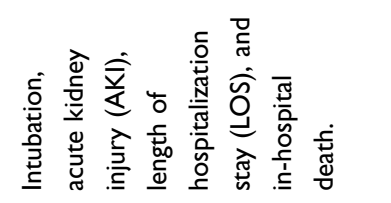 & $\stackrel{\frac{a}{1}}{\varrho}$ \\
\hline $\begin{array}{l}\sum_{a}^{T} \\
\stackrel{I}{I} \\
\pm\end{array}$ & 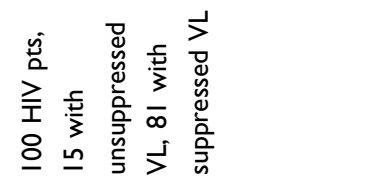 & $\sum_{\substack{D \\
\infty}}^{T}$ \\
\hline 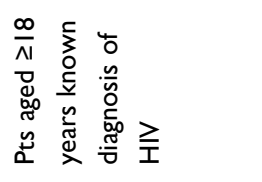 & 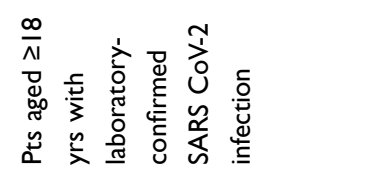 & 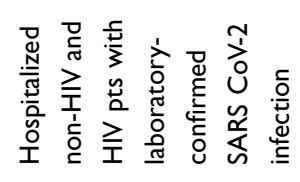 \\
\hline 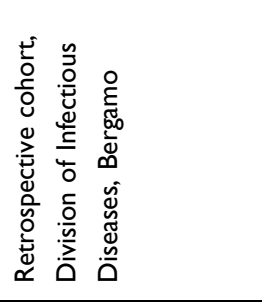 & 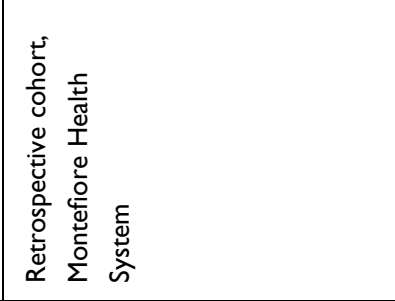 & 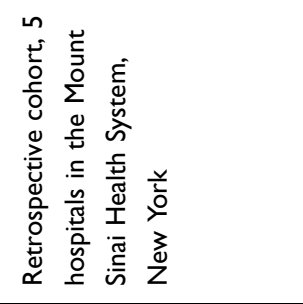 \\
\hline 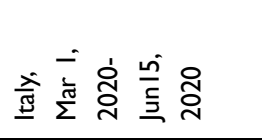 & 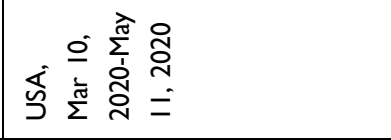 & 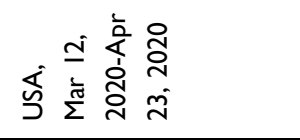 \\
\hline 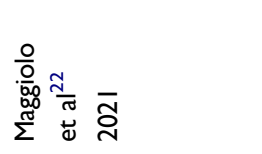 & 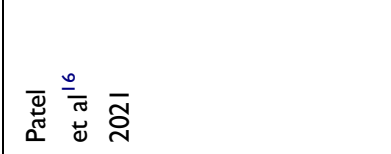 & 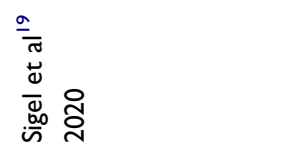 \\
\hline
\end{tabular}




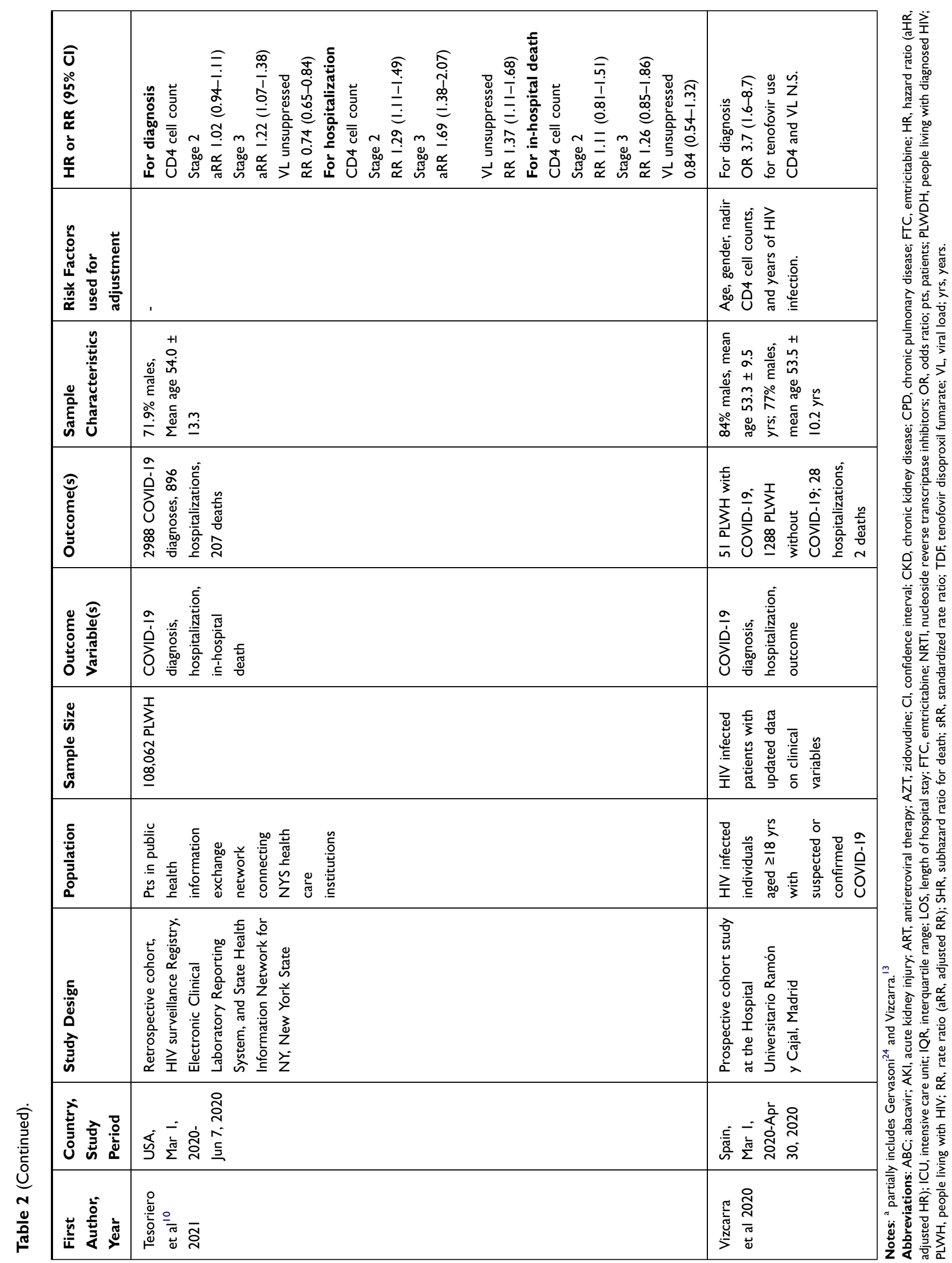


disease, chronic kidney disease, race, nicotine dependence and sex.

In their observational prospective study, Vizcarra et $\mathrm{al}^{14}$ observed a high hospitalization rate $(55 \%)$, but their sample was small (51 patients).

A retrospective observational study conducted by Cabello et $\mathrm{al}^{11}$ analyzed 63 HIV-positive patients with COVID-19, observing a lower hospitalization rate in PLWH confirmed cases compared with PNLWH (48.4\% vs $63.5 \%$ ).

Braunstein et $\mathrm{al}^{12}$ demonstrated that, in comparison with all diagnosed COVID-19 cases in NYC, a higher proportion of PLWH were hospitalized for COVID-19 (42\% vs $26 \%)$.

\section{HIV Infection and ICU Admission for COVID-19}

In the first three months of the SARS-CoV-2 infection outbreak in NYC, Braunstein et $\mathrm{al}^{12}$ showed a rate of ICU admission of 5\% in 2410 PLWH vs $3 \%$ in 202,012 PNLWH with confirmed COVID-19.

Patel et al $^{16}$ conducted a retrospective cohort study on 4613 COVID-19 positive patients; 100 were PLWH. In an analysis adjusted for age, sex, race/ethnicity, BMI, history of chronic lower respiratory diseases and calendar time, PLWH had an increased risk of intubation compared to PNLWH (aHR 1.73, 95\% CI 1.12-2.67; $\mathrm{p}=0.01$ ).

Miyashita et al ${ }^{17}$ also carried out a retrospective study on 8912 patients with COVID-19; 161 had also HIV infection. The author observed a higher risk of intubation in the age group under 50 years (relative risk 2.97, 95\% CI, 1.29-6.84), whilst no differences were observed in the other age groups.

On the contrary, Geretti et $\mathrm{al}^{9}$ reported no differences between HIV-negative patients and PLWH in the risk of admission to ICU, after adjustment for sex, ethnicity, age, baseline date, indeterminate/probable hospital acquisition of COVID-19 and comorbidities, regardless of HIV status (odds ratio, OR 1.22, 95\% CI, 0.80-1.87; $\mathrm{p}=0.35$ ).

\section{HIV Infection and Mortality for COVID-19}

A very large retrospective cohort study, reported by Bhaskaran et al $^{18}$ compared the risk of COVID-19 death among PLWH and PNLWH: crude mortality risk was similar between the two groups (HR 1.03, 95\% CI $0.7-$ 1.52); after adjusting for age and sex, HIV infection was associated with a higher risk of COVID-19 death (HR $2.90,95 \%$ CI 1.96-4.30; $p<0.0001$ ), with a slight attenuation after further adjustment for index of multiple deprivation, ethnicity, obesity and smoking (HR 2.59, 95\% CI, 1.74-3.84; p $<0.0001$ ).

The Western Cape Department of Health (South Africa) performed a study on 223,080 COVID-19 positive patients $^{15}$, 625 of them died. A greater proportion of COVID-19 death was observed in PLWH younger than 50 years compared with same age PNLWH (39\% vs 13\%). After adjusting for age, sex, and other comorbidities, HIV was associated with increased COVID-19 mortality (aHR 2.14, 95\% CI 1.70-2.70), irrespectively of viremia or immunosuppression prior to the COVID-19 infection.

In the large Tesoriero cohort study ${ }^{10}$ (2988 patients), no differences were observed in in-hospital death between people living with diagnosed HIV infection, and people without diagnosed HIV infection (adjusted rate ratio 0.98, 95\% CI 0.85-1.12). Anyway, a significantly higher mortality rate per person and per diagnosis was observed in PLWH (case fatality rate, 69.28 per 1000 vs 38.70 per 1000: sRR 1.30, 95\% CI, 1.13-1.43).

Hadi et $\mathrm{al}^{8}$ performed an analysis on all patients diagnosed with COVID-19 (total 50,167), who were divided into two cohorts, based on the presence or absence of HIV infection: 49,763 patients without concurrent HIV infection and 404 with preexisting HIV diagnosis. In the unmatched analysis, PLWH had higher mortality at 30 days from COVID-19 diagnosis (4.95\% vs 3.2\%, risk ratio 1.55, 95\% CI, 1.01-2.39), while after matching for BMI, diabetes, hypertension, chronic lung diseases, chronic kidney diseases, race, nicotine dependence and sex, no difference in mortality was found between the two groups $(5.0 \%$ vs $3.7 \%$, risk ratio 1.33 , $95 \% \mathrm{CI}$, $0.69-2.57 \%$ ). These observations are probably due to the demographic characteristics of PLWH (greater proportion of men, African American race, obesity, and the other listed comorbidities). No differences in mortality were found even in two subgroup analyses, including only (1) patients with HIV-associated diseases (risk ratio 1.12, 95\% CI 0.59-2.12) or (2) patients with antiretroviral therapy history (risk ratio $1.22,95 \%$ CI $0.68-2.18$ ), compared to non-HIV subjects.

On the other hand, Braunstein et $\mathrm{al}^{12}$ reported a mortality of $13 \%$ vs $8 \%$ in PLWH vs PNLWH.

The retrospective observational study that was conducted by Cabello et al ${ }^{11}$ observed a lower global mortality rate in PLWH (3.22\% vs $13.3 \%)$, including severe cases $(6.7 \%$ vs $21.0 \%)$. No association was observed between HIV-related factors and COVID-19 severity. 
Considering studies that enrolled less than 200 PLWH, most did not report higher mortality in the overall PLWH population (Table 1), ${ }^{9,16,17,19}$ whereas some differences were observed in patients aged less than 60 years in the study of Geretti et $\mathrm{al}^{9}$ and in patients aged less than 50 years in the study of Miyashita et al. ${ }^{17}$

\section{Risk Factors for COVID-19 Severity in PLWH Risk Factors for Severe COVID-19}

In PLWH, rates of severe COVID-19 ranged from 11\% to $28 \%$ (Table 2). ${ }^{7,14,19-22}$ Severe SARS-CoV-2 Infection was associated mostly with age and presence of comorbidities (see Table 3).

In a cohort of $175 \mathrm{PLWH}$, Hoffman et $\mathrm{al}^{20}$ reported a $28 \%$ rate of severe COVID-19 (49/175). Severity of COVID-19 was associated with age equal or higher than 50 years (OR 2.49, 95\% CI 1.24-5.03), nadir CD4 $<200$ cells $/ \mu \mathrm{L}$ (OR 2.10, 95\% CI 1.05-4.21) and current CD4 $<350$ cells $/ \mu \mathrm{L}$ (OR 3.30, 95\% CI 1.49-7.31).

On the other hand, Inciarte et $\mathrm{al}^{7}$ showed a severity rate of $11.3 \%(6 / 53)$ in a cohort of PLWH with a median age of 44 years (interquartile range, IQR, 36-52), nadir CD4 303 (IQR 140-434) and last CD4 624 (IQR 462-838) with a $43 \%$ prevalence of comorbidities (23/43, mainly hypertension and diabetes). No association was found with immune-virological parameters and anti-retroviral treatment.

In most studies, patients had high CD4 (usually $>500$ cells $/ \mu \mathrm{L}$ ) and suppressed HIV-viral load (VL).

\section{Hospital Admission}

The reported hospitalization rates ranged from $27 \%$ to $63 \%$ (Table 2). ${ }^{10,23-26}$ Age, low CD4 nadir and low current CD4 were the most frequent risk factors.

In Italy, Gervasoni et $\mathrm{al}^{24}$ showed a hospitalization rate of $27.7 \%$ (13/47) in PLWH with mean CD4 of $636 \pm 290$ cells $/ \mu \mathrm{L}$, mostly with undetectable HIV-VL $(94 \%<20$ copies $/ \mathrm{mL}$ ) with mean age of $51 \pm 11$ years. Di Biagio et $\mathrm{al}^{25}$ showed a higher rate of hospitalization in PLWH with lower nadir CD4 and lymphocytes.

Del Amo et $\mathrm{al}^{26}$ described a hospitalization rate of $63.9 \%(151 / 236)$, with a higher risk for men and subjects older than 70 years, and a lower risk in patients receiving tenofovir/emtricitabine.

Two large cohorts ${ }^{10,15}$ described hospitalization rates lower than $30 \%$.

Tesoriero et al ${ }^{10}$ showed a hospitalization rate of $30 \%$ in 2988 PLWH in New York State, with a higher risk in patients with lower CD4 and unsuppressed VL, while Boulle et $\mathrm{a}^{15}$ described a low percentage of hospital admission in PLWH $(601 / 3978,15.1 \%)$ in South Africa, with a higher risk in patients with low CD4. These significant differences could be due to different socioeconomic status and to significantly lower age in Boulle's study. However, lower CD4 were consistently reported as a risk factor for hospitalization in PLWH.

\section{ICU Admission}

Rates of ICU admission ranged from $5 \%$ to $28.7 \%$ (Table 2).

Dandachi et $\mathrm{al}^{23}$ showed an ICU admission rate of $28.7 \%$ (47/286) in a sample of PLWH aged 51.4 years on average, with a higher risk in patients with $\mathrm{CD} 4<200$ cells $/ \mu \mathrm{L}$.

Ho et $\mathrm{al}^{27}$ reported an ICU admission rate of $26.4 \%$ (19/72) in patients with median CD4 554 (IQR 339-752) and HIV-VL $<50$ copies $/ \mathrm{mL}$ at last visit. The median age was 58 years (IQR 52-65).

Patel et al ${ }^{16}$ showed a risk of intubation in $26 \%(21 / 81)$ of PLWH, independently of HIV-VL suppression, in a sample with median age of 65 years (IQR 54-76). Moreover, in this study, increased CD4 were related with an increased risk of intubation (aHR 1.13, 95\% CI 10.61.20 , by 100 cells $/ \mathrm{mmc}$ increase in CD4).

On the other hand, Braunstein et $\mathrm{al}^{12}$ described a $5 \%$ rate of ICU admission, with a higher risk in patients with CD4 less than 200 cells $/ \mu \mathrm{L}$.

Vizcarra et $\mathrm{al}^{14}$ described an ICU admission rate of $12 \%(6 / 51)$ in a cohort of PLWH with a mean age of 53.3 $( \pm 9.5)$ years, with $63 \%$ of patients affected from at least one comorbidity, median CD4 nadir 224 (IQR 101-437), recent CD4 565 (IQR 296-782) and HIV-VL $<50$ copies in $98 \%$ of patients.

\section{Death}

The reported mortality rates are extremely variable, depending on sample characteristics, region of origin and socio-economic factors (Table 2).

Evaluating HIV-related risk factors, Boulle et $\mathrm{al}^{15}$ showed data about 199 hospitalized patients with available CD4: 70 had CD4 $<200$ cells $/ \mu \mathrm{L}$. In this group, a higher mortality was demonstrated (aHR 1.97, 95\% CI 1.14-3.40) in comparison to PLWH with CD4 $\geq 350$ cells $/ \mu \mathrm{L}$. A protective role of tenofovir disoproxil-fumarate vs abacavir was also found (aHR 0.41, 95\% CI 0.21-0.78).

Dandachi et al $^{23}$ described a sample of PLWH mostly $(95 \%)$ on antiretroviral therapy (ART) and with viral 


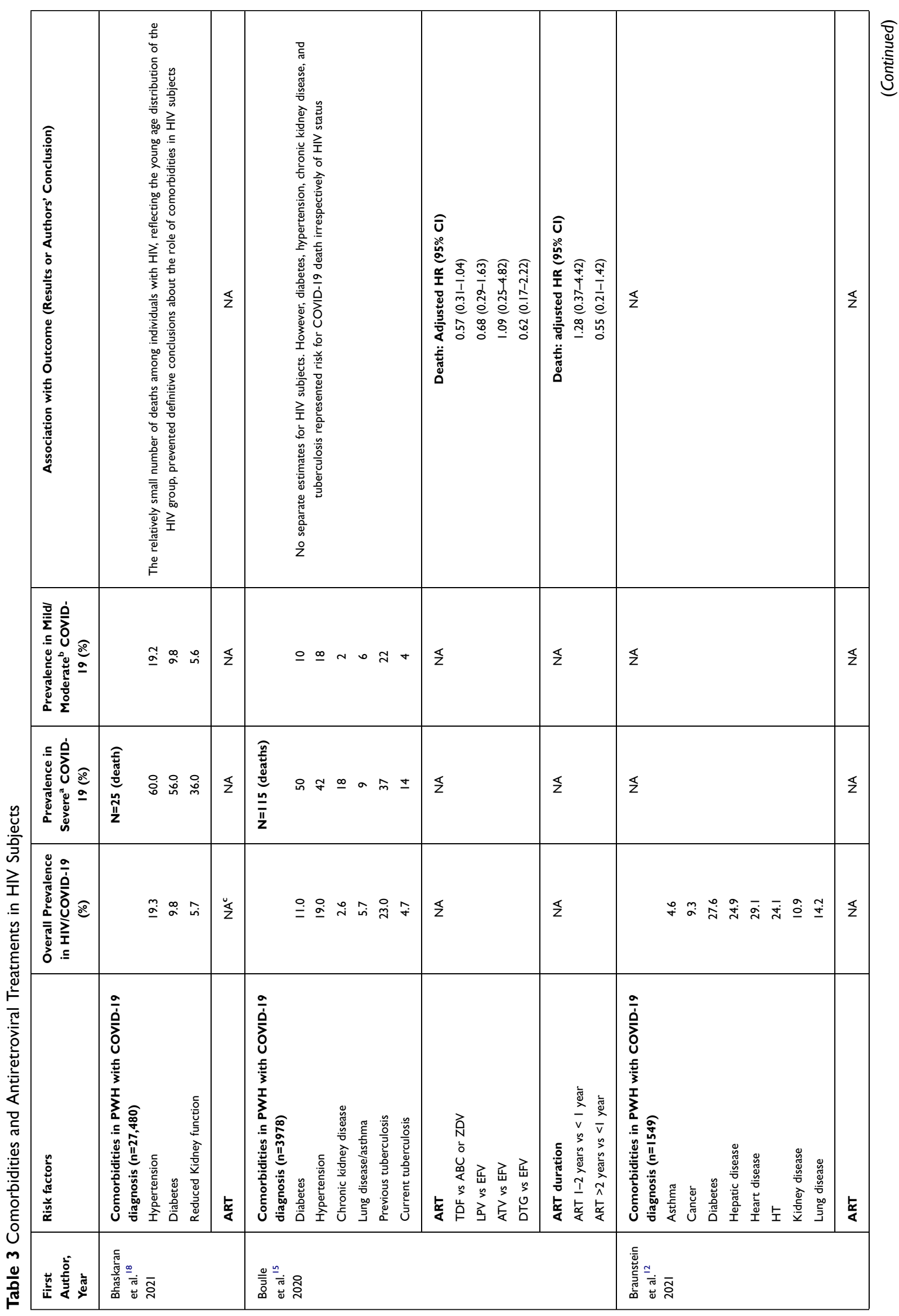




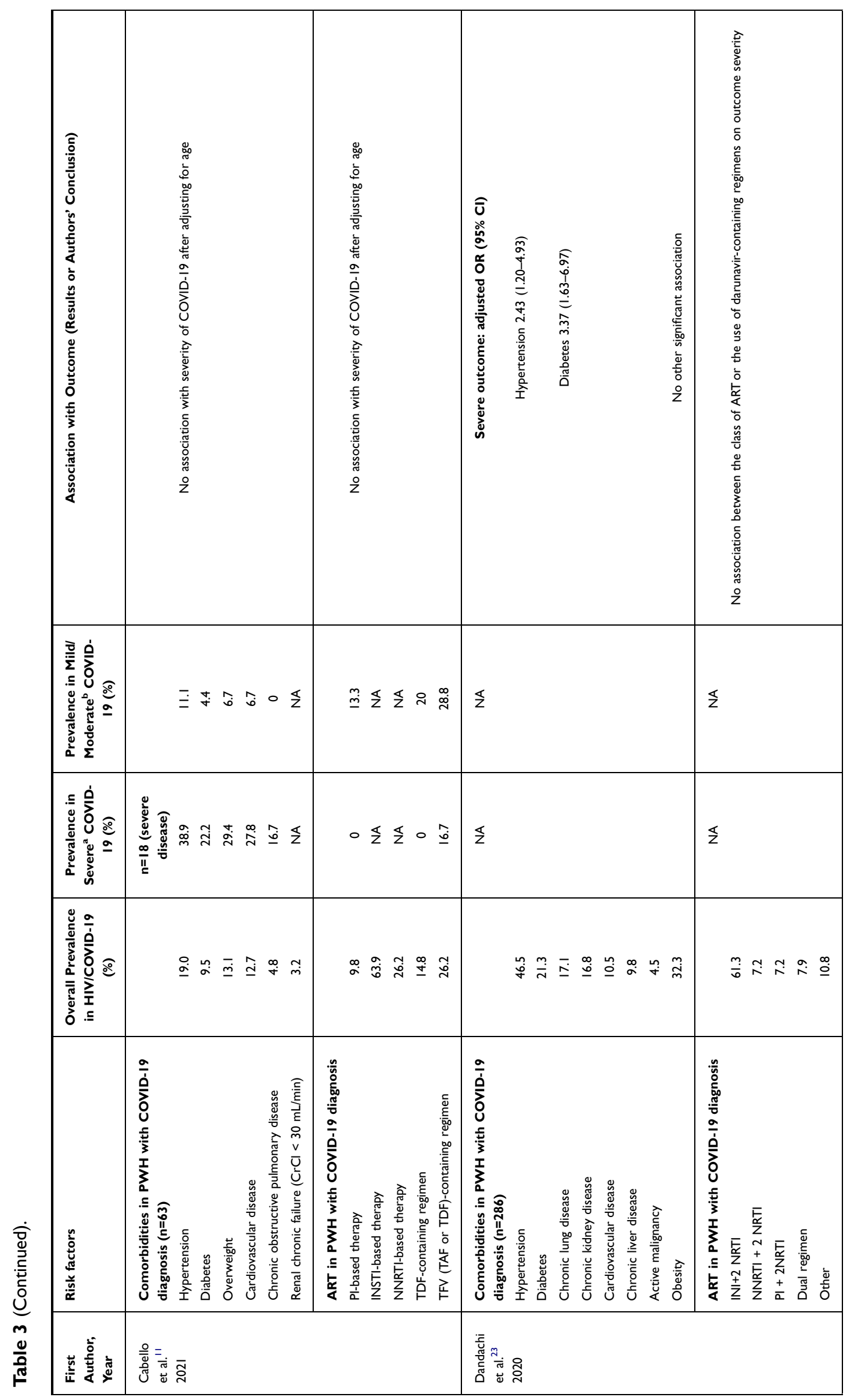




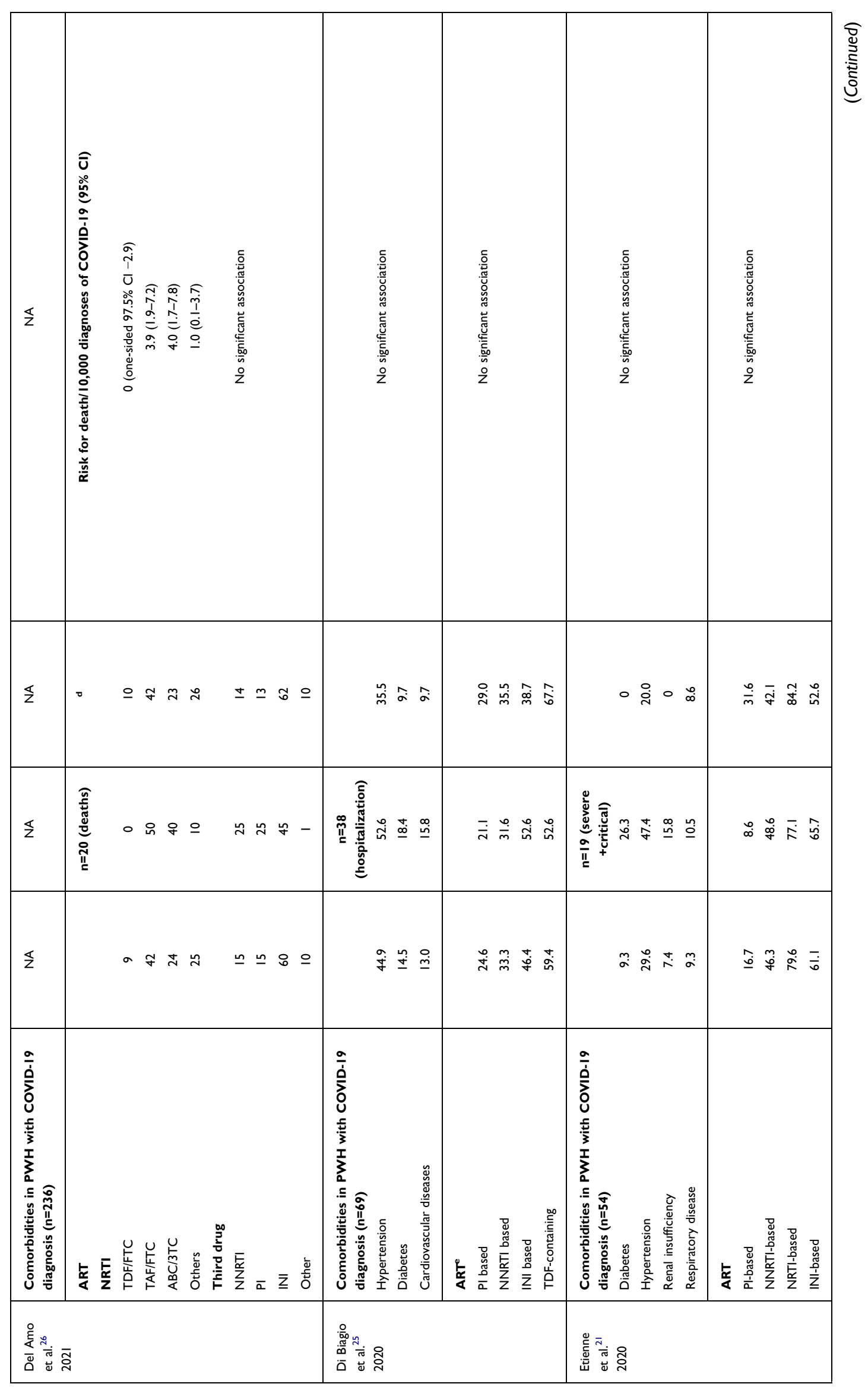




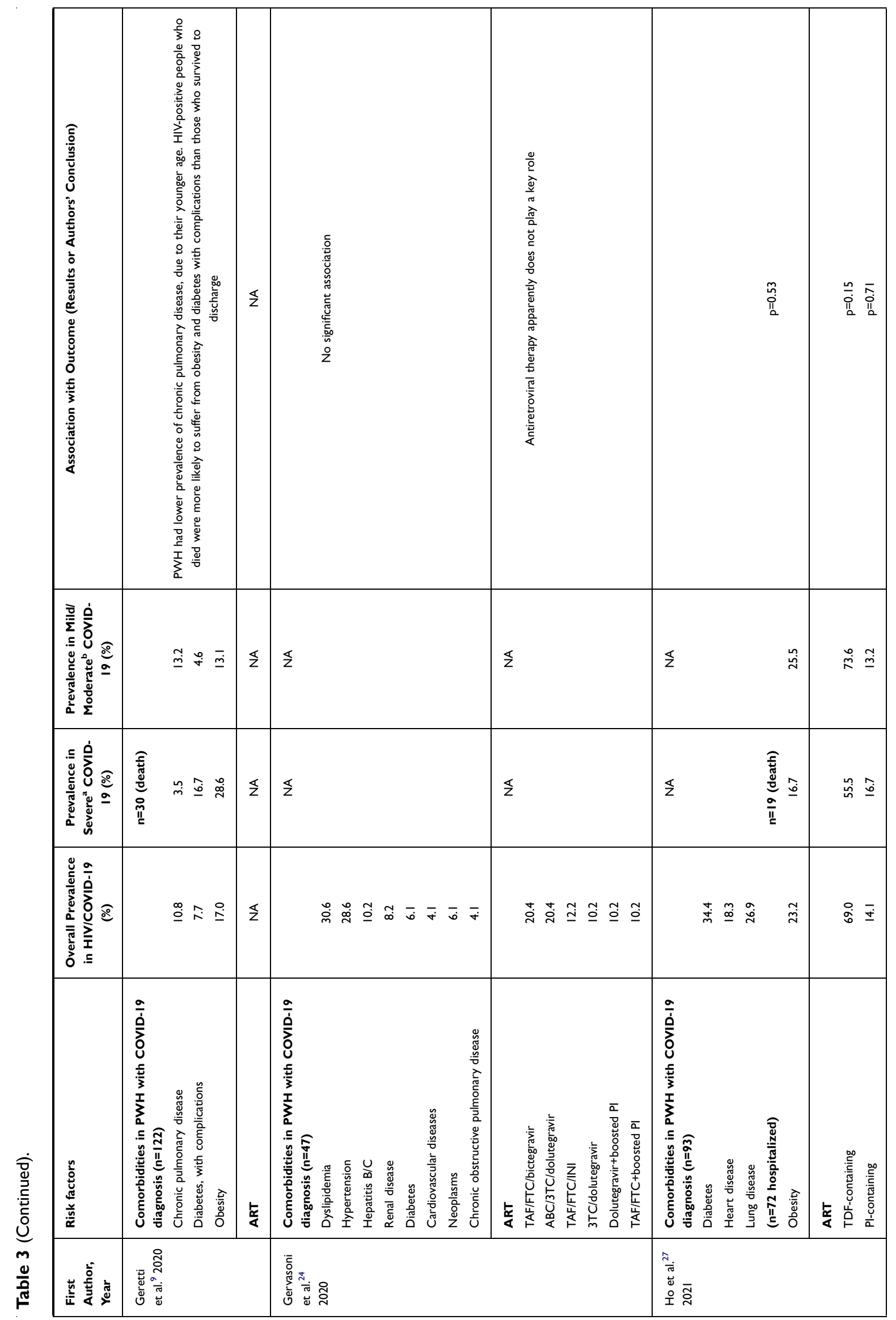


$\underline{\text { Dovepress }}$

Squillace et al

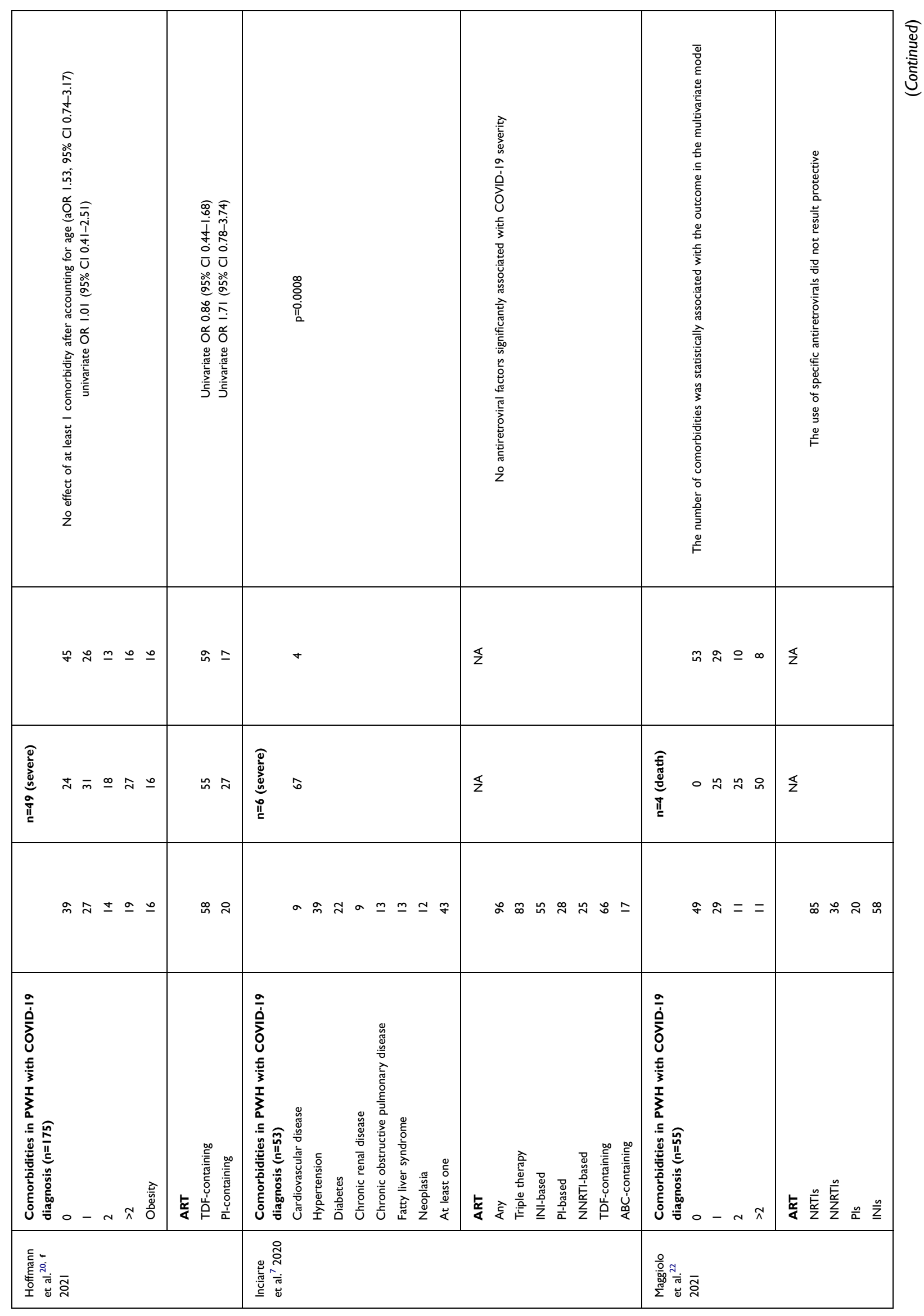

Infection and Drug Resistance 2021:14

DovePress

4009 


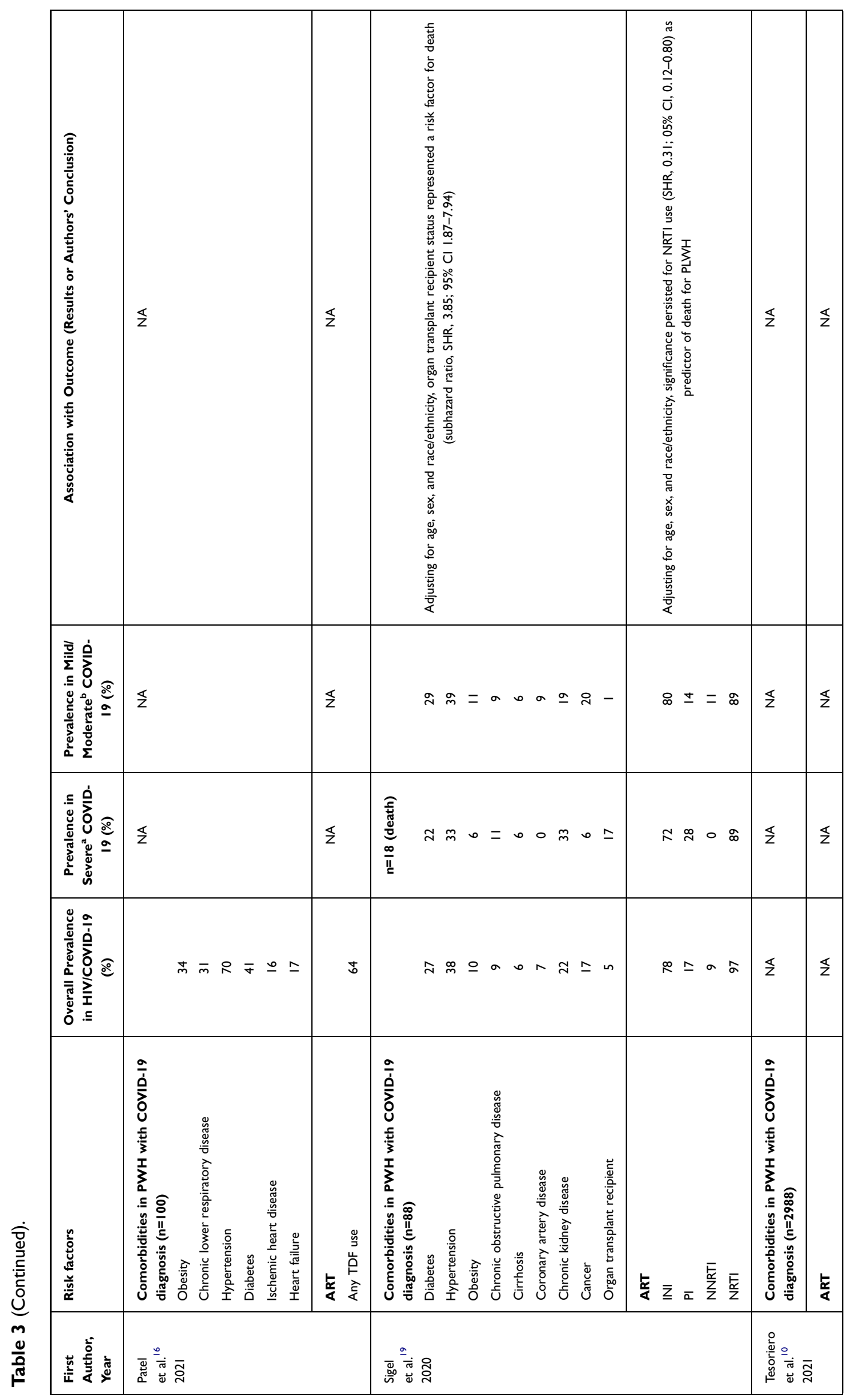




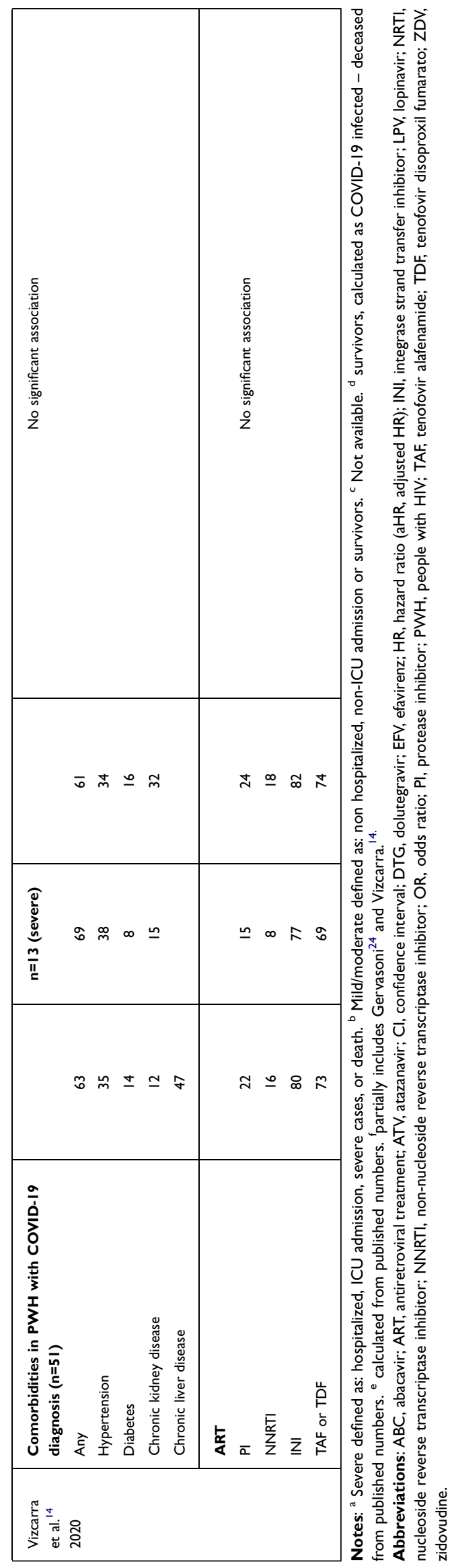

suppression (88.7\%). At the multivariable analysis, CD4<200 cells $/ \mu \mathrm{L}$ (OR 3.32, 95\% CI 1.11-9.93), chronic lung disease (OR 3.65, 95\% CI 1.56-8.56) and three or more comorbidities (OR 5.09, 95\% CI 1.05-24.76) were associated with a severe outcome (composite endpoint including ICU admission, use of mechanical ventilation and death).

Geretti et $\mathrm{al}^{9}$ reported that age (OR 1.05, 95\% CI 1.011.09), diabetes (OR 2.18, 95\% CI 1.02-4.66) and obesity (OR 2.89, 95\% CI 1.28-6.53) were risk factors for SARSCoV-2-related mortality, at multivariable analysis.

$\mathrm{Ho}$ et $\mathrm{al}^{27}$ reported that a lower lymphocyte nadir was significantly associated with death in a sample of PLWH with median CD4 554 (IQR 339-752) and HIV-VL $<50$ copies/ $\mathrm{mL}$ at the last visit, with a median age of 58 (IQR 52-65).

Sigel et $\mathrm{al}^{19}$ described a cohort of PLWH with a median age of 61 years (IQR 54-67) with $44 \%$ of patients with $\mathrm{CD} 4>500$ cells $/ \mu \mathrm{L}$ and $81 \%$ with undetectable HIV.

In the multivariate analysis, organ transplant recipient status was a risk factor for death (sub-hazard ratio, SHR, 3.85 , 95\% CI 1.87-7.94), while nucleoside reverse transcriptase inhibitors (NRTI) use was a protective factor (SHR 0.31, 95\% CI 0.12-0.80).

Patel et $\mathrm{al}^{16}$ described a $22 \%$ death rate $(22 / 100)$ in a cohort of HIV patients, without any significant differences between the group with suppressed HIV-VL vs unsuppressed HIV-VL.

Smaller studies usually reported a mortality rate $\leq 10 \%,{ }^{14,22,24,26}$ showing older age and comorbidities as risk factors. In these studies, most patients were on ART, had CD4>500 cells $/ \mu \mathrm{L}$ and undetectable HIV-VL.

\section{Discussion}

In most studies, HIV infection did not represent a risk factor for SARS-CoV-2 infection.

Evidence from larger studies ${ }^{7,10-12}$ did not support a higher risk of SARS-CoV-2 infection in PLWH.

Only Sachdev et $\mathrm{al}^{13}$ described a higher risk of SARSCoV-2 in PLWH than in PNLWH, but $45 \%$ of PLWH in their sample experienced unstable housing, a factor that could have influenced the rate of infection. In the study by Tesoriero et al, ${ }^{10}$ a higher risk of SARS-CoV-2 diagnosis was reported in older age, black race, and Hispanic individuals, with a probable link with socioeconomic status rather than HIV infection per se.

So far, in the published literature, data were scarce and inconsistent about the effect of ART regimen on the chance of SARS-CoV-2 infection and clinical outcome of 
COVID-19 in PLWH. Large studies are needed to confirm or exclude the role of ART.

Del Amo et $\mathrm{al}^{26}$ and Boulle et $\mathrm{al}^{15}$ found a lower risk of infection in patients on tenofovir therapy, suggesting a possible inhibiting effect on RNAdRNAp of SARS-CoV $-2,{ }^{28}$ whereas Sigel et al ${ }^{19}$ observed a lower risk in PLWH on current NRTI therapy, but did not differentiate among individual drugs.

The protective role of tenofovir is a matter of debate, with conflicting evidence about its possible effect on COVID-19 prevention and treatment. ${ }^{4}$

Protease inhibitors, which had a role in treating the MERS-CoV infection, did not seem effective in preventing or treating SARS-CoV-2. ${ }^{29}$

Once SARS-CoV-2 infection is acquired, risk of severe COVID-19 and hospitalization is higher in PLWH. Low CD4 are consistently reported as a risk factor for severe COVID-19. Tesoriero et $\mathrm{al}^{10}$ demonstrated an increasing risk of hospitalization across the three stages of HIV infection (CD4 $\geq 500$ vs $200-499$ vs $<200$ cells $/ \mu \mathrm{L}$ ). Age older than 50 years and the presence of comorbidities are other strong risk factors for the disease severity. Uncontrolled HIV-VL was not reported as a risk factor for COVID-19 severity in three studies, ${ }^{15,16,23}$ although PLWH with uncontrolled VL represented less than $15 \%$ of enrolled patients.

Few data are available on PLWH younger than 50 years, which are underrepresented in the studies on COVID-19 severity.

Inciarte et $\mathrm{al}^{7}$ described a low prevalence of severe SARS-CoV-2 infection in a population mostly younger than 50 years.

Many studies ${ }^{14,19,21,22}$ reporting a prevalence of severe cases higher than $20 \%$ showed a median age between 52 and 61 .

From the published evidence, PLWH aged 50 or more years appear more at risk of severe COVID-19, especially in the presence of severe immune suppression $(C D 4<200$ cells/micrL). This could be due both to the higher burden and early development of comorbidities in this group of $\mathrm{PLWH}^{2,30}$ and to the immune dysfunction occurring in PLWH with $\mathrm{CD} 4<200$ cells $/ \mu \mathrm{L}$.

Low CD4 have been also associated with poor outcomes in the general population. ${ }^{31}$

We can hypothesize that CD4 at diagnosis of SARSCoV-2 infection were extremely low as a consequence of SARS-CoV-2 infection. However, in most studies, CD4 during the course of COVID-19 were not available.
"Current CD4" were usually defined as retrospectively collected, from three years to four weeks before SARSCOV-2 infection diagnosis. Only Patel et $\mathrm{al}^{16}$ stated that they included CD4 during hospitalization only when a previous measure was unavailable; nonetheless, they did not stratify their results according to time of CD4 measurement. Thus, we were unable to evaluate the possible role of fast CD4 loss during SARS-CoV-2 infection.

However, there are several reasons for inappropriate immune response in HIV infection.

A prompt and hard CD4 response has been reported to be necessary for IgG and IgA response to SARS-CoV-2. ${ }^{32}$

Immune activation has been demonstrated to persist in HIV chronic infection with alteration in kynureninetryptophan ratio, a marker of defective adaptive immunity, even in patients treated early for HIV infection..$^{33,34}$

On the other hand, HIV and SARS-CoV-2 share a common evasion of innate immunity, ${ }^{35}$ that could explain a deficient response to Interferon.

Inflammation and immune-activation related to severe HIV infection (CD4 <200) could explain the worse prognosis of SARS-CoV-2 infection in this subset of patients.

The risk of ICU admission was reported as higher than in people not living with HIV in most studies. ${ }^{12,16,17} \mathrm{CD} 4$ $<200$ were a risk factor for ICU admission in the study of Dandachi et $\mathrm{al}^{23}$ while the increase in current CD4 (per 100 cells $/ \mu \mathrm{L}$ ) was a risk factor for Patel et al. ${ }^{16}$ The two studies included a slightly different number of intubated patients with significantly different ages: Dandachi et al enrolled 47 intubated patients (twice as much than Patel et al) with significantly younger age. This difference could partially explain the inconsistency of their findings. The immune reconstitution as a risk factor could be an interesting hypothesis in the development of cytokine storm in COVID-19, but high CD4 were not frequently associated with severity of disease, even if most patients enrolled in these studies had CD4 $>500$ cells $/ \mu \mathrm{L}$ and suppressed HIVVL. However, as previously discussed, low and dysfunctional CD4 are not the only immune dysfunction in HIV infection.

PLWH with high CD4 (>500) did not show a higher risk of cytokine storm in most studies, suggesting that, in this group of patients, the role of comorbidities could be stronger than that of CD4.

In PLWH, higher mortality rates were reported in two large studies. ${ }^{12,15}$

It is interesting that in the study of Tesoriero et $\mathrm{al}^{10}$ they did not find a significantly higher mortality rate in 
PLWH per hospitalization, but when they analyzed per population and per diagnosis, HIV infection represented a risk factor for death. These results were likely due to the higher percentage of PLWH with severe disease.

On the other hand, Hadi et $\mathrm{al}^{8}$ demonstrated a higher mortality in PLWH but adjusting for BMI, diabetes, hypertension, chronic lung diseases, chronic kidney diseases, race, nicotine dependence and sex, no differences were found between PLWH vs PNLWH.

$\mathrm{CD} 4<200$ cells $/ \mu \mathrm{L}$, age and comorbidities were the most documented risk factors for mortality in PLWH.

The role of comorbidities has been demonstrated to be crucial. In particular, in Miyashita's study ${ }^{17}$ most PLWH who deceased younger than 50 years old were male, with hypertension and other comorbidities. In the study by Geretti et al, ${ }^{9}$ a higher risk of mortality was also reported in PLWH aged $<60$ years, especially if associated with obesity and diabetes.

Besides median age and number of comorbidities that are increasing in cohorts of PLWH, especially in Europe, ${ }^{36}$ another concern is that chronic airway abnormalities and pulmonary inflammation are highly prevalent in HIV infection, even in non-smokers. ${ }^{37,38}$

This review has some limitations. First, we did not perform a meta-analysis and we cannot evaluate the impact of statistical power in the selected studies. Secondly, we did not evaluate all the studies on HIV and SARS-CoV-2 but only those including more than 40 PLWH.

In conclusion, HIV infection did not seem a risk factor for SARS-CoV-2 infection, but when PLWH acquire COVID-19 a worse outcome was more frequent than in PNLWH, especially in case of severe immune deficiency $(\mathrm{CD} 4<200)$ and in presence of multiple comorbidities.

\section{Disclosure}

Dr Nicola Squillace reports personal fees from ViiV Healthcare, outside the submitted work. The authors report no other conflicts of interest in this work.

\section{References}

1. Docherty AB, Harrison EM, Green CA, et al. Features of 20133 UK patients in hospital with covid-19 using the ISARIC WHO clinical characterisation protocol: prospective observational cohort study. BMJ. 2020;369:m1985. doi:10.1136/bmj.m1985

2. So-Armah K, Benjamin LA, Bloomfield GS, et al. HIV and cardiovascular disease. Lancet HIV. 2020;7(4):e279-e293. doi:10.1016/S23523018(20)30036-9

3. Peng X, Ouyang J, Isnard S, et al. Sharing CD4+ T cell loss: when COVID-19 and HIV collide on immune system. Front Immunol. 2020;11:596631. doi:10.3389/fimmu.2020.596631
4. Zanella I, Zizioli D, Castelli F, Quiros-Roldan E. Tenofovir, another inexpensive, well-known and widely available old drug repurposed for SARS-COV-2 infection. Pharmaceuticals. 2021;14(5):454. doi: $10.3390 /$ ph14050454

5. Ambrosioni J, Blanco JL, Reyes-Uruena JM, et al. Overview of SARS-CoV-2 infection in adults living with HIV. Lancet HIV. 2021;8(5):e294-e305. doi:10.1016/S2352-3018(21)00070-9

6. Maggi P, Ricci E, Messina V, et al. Dangerous liaisons? The role of inflammation and comorbidities in HIV and SARS-CoV-2 infection. Expert Rev Clin Immunol. 2021;17(3):201-208. doi:10.1080/ 1744666X.2021.1886080

7. Inciarte A, Gonzalez-Cordon A, Rojas J, et al. Clinical characteristics, risk factors, and incidence of symptomatic coronavirus disease 2019 in a large cohort of adults living with HIV: a single-center, prospective observational study. AIDS. 2020;34(12):1775-1780. doi:10.1097/QAD.0000000000002643

8. Hadi YB, Naqvi SFZ, Kupec JT, Sarwari AR. Characteristics and outcomes of COVID-19 in patients with HIV: a multicentre research network study. AIDS. 2020;34(13):F3-F8. doi:10.1097/QAD.0000000000002666

9. Geretti AM, Stockdale AJ, Kelly SH, et al. Outcomes of COVID-19 related hospitalization among people with HIV in the ISARIC WHO clinical characterization protocol (UK): a prospective observational study. Clin Infect Dis. 2020. Available from: https://academic.oup.com/cid/advance-article/doi/ 10.1093/cid/ciaa1605/5937133. Accessed September 18, 2021.

10. Tesoriero JM, Swain CE, Pierce JL, et al. COVID-19 outcomes among persons living with or without diagnosed HIV infection in New York state. JAMA Netw Open. 2021;4(2):e2037069. doi:10.1001/jamanetworkopen.2020.37069

11. Cabello A, Zamarro B, Nistal S, et al. COVID-19 in people living with HIV: a multicenter case-series study. Int $J$ Infect Dis. 2021;102:310-315. doi:10.1016/j.ijid.2020.10.060

12. Braunstein SL, Lazar R, Wahnich A, Daskalakis DC, Blackstock OJ. Coronavirus disease 2019 (COVID-19) infection among people with human immunodeficiency virus in new york city: a population-level analysis of linked surveillance data. Clin Infect Dis. 2021;72(12): e1021-e1029. doi:10.1093/cid/ciaa1793

13. Sachdev D, Mara E, Hsu L, et al. COVID-19 susceptibility and outcomes among people living with HIV in San Francisco. J Acquir Immune Defic Syndr. 2021;86(1):19-21. doi:10.1097/ QAI.0000000000002531

14. Vizcarra P, Perez-Elias MJ, Quereda C, et al. Description of COVID-19 in HIV-infected individuals: a single-centre, prospective cohort. Lancet HIV. 2020;7(8):e554-e564. doi:10.1016/S23523018(20)30164-8

15. Boulle A, Davies MA, Hussey H, et al. Risk factors for COVID-19 death in a population cohort study from the Western Cape Province, South Africa. Clin Infect Dis. 2020. doi: 10.1093/cid/ciaa1198

16. Patel VV, Felsen UR, Fisher M, et al. Clinical outcomes and inflammatory markers by HIV serostatus and viral suppression in a large cohort of patients hospitalized with COVID-19. J Acquir Immune Defic Syndr. 2021;86(2):224-230. doi:10.1097/QAI.0000000 000002578

17. Miyashita H, Kuno T. Prognosis of coronavirus disease 2019 (COVID-19) in patients with HIV infection in New York city. HIV Med. 2021;22(1):e1-e2. doi:10.1111/hiv.12920

18. Bhaskaran K, Rentsch CT, MacKenna B, et al. HIV infection and COVID-19 death: a population-based cohort analysis of UK primary care data and linked national death registrations within the openSAFELY platform. Lancet HIV. 2021;8(1):e24-e32. doi:10.1016/S2352-3018(20)30305-2

19. Sigel K, Swartz T, Golden E, et al. Coronavirus 2019 and people living with human immunodeficiency virus: outcomes for hospitalized patients in New York City. Clin Infect Dis. 2020;71 (11):2933-2938. doi:10.1093/cid/ciaa880 
20. Hoffmann C, Casado JL, Harter G, et al. Immune deficiency is a risk factor for severe COVID-19 in people living with HIV. HIV Med. 2021;22(5):372-378. doi:10.1111/hiv.13037

21. Etienne N, Karmochkine M, Slama L, et al. HIV infection and COVID-19: risk factors for severe disease. AIDS. 2020;34 (12):1771-1774. doi:10.1097/QAD.0000000000002651

22. Maggiolo F, Zoboli F, Arosio M, et al. SARS-CoV-2 infection in persons living with HIV: a single center prospective cohort. $J$ Med Virol. 2021;93(2):1145-1149. doi:10.1002/jmv.26352

23. Dandachi D, Geiger G, Montgomery MW, et al. Characteristics, comorbidities, and outcomes in a multicenter registry of patients with HIV and coronavirus disease-19. Clin Infect Dis. 2020. doi: $10.1093 / \mathrm{cid} / \mathrm{ciaa} 1339$

24. Gervasoni C, Meraviglia P, Riva A, et al. Clinical features and outcomes of patients with human immunodeficiency virus with COVID-19. Clin Infect Dis. 2020;71(16):2276-2278. doi:10.1093/ $\mathrm{cid} / \mathrm{ciaa} 579$

25. Di Biagio A, Ricci E, Calza L, et al. Factors associated with hospital admission for COVID-19 in HIV patients. AIDS. 2020;34 (13):1983-1985. doi:10.1097/QAD.0000000000002663

26. Del Amo J, Polo R, Moreno S, et al. Incidence and severity of COVID-19 in HIV-positive persons receiving antiretroviral therapy: a cohort study. Ann Intern Med. 2020;173(7):536-541. doi:10.7326/ M20-3689

27. Ho HE, Peluso MJ, Margus C, et al. Clinical outcomes and immunologic characteristics of coronavirus disease 2019 in people with human immunodeficiency virus. J Infect Dis. 2021;223(3):403-408. doi:10.1093/infdis/jiaa380

28. Elfiky AA. Ribavirin, Remdesivir, Sofosbuvir, Galidesivir, and Tenofovir against SARS-CoV-2 RNA dependent RNA polymerase (RdRp): a molecular docking study. Life Sci. 2020;253:117592. doi:10.1016/j.lfs.2020.117592

29. Vargas M, Servillo G, Einav S. Lopinavir/ritonavir for the treatment of SARS, MERS and COVID-19: a systematic review. Eur Rev Med Pharmacol Sci. 2020;24(16):8592-8605.
30. Guaraldi G, Orlando G, Zona S, et al. Premature age-related comorbidities among HIV-infected persons compared with the general population. Clin Infect Dis. 2011;53(11):1120-1126. doi:10.1093/ cid/cir627

31. Zhang $\mathrm{H}, \mathrm{Wu}$ T. CD4+T, CD8+T counts and severe COVID-19: a meta-analysis. $J$ Infect. 2020;81(3):e82-e84. doi:10.1016/j. jinf.2020.06.036

32. Grifoni A, Weiskopf D, Ramirez SI, et al. Targets of T cell responses to SARS-CoV-2 coronavirus in humans with COVID-19 disease and unexposed individuals. Cell. 2020;181(7):1489-1501. e1415. doi:10.1016/j.cell.2020.05.015

33. Schnittman SR, Deitchman AN, Beck-Engeser G, et al. Abnormal levels of some biomarkers of immune activation despite very early treatment of human immunodeficiency virus. J Infect Dis. 2021;223 (9):1621-1630. doi:10.1093/infdis/jiaa580

34. Routy JP, Mehraj V, Vyboh K, Cao W, Kema I, Jenabian MA. Clinical relevance of kynurenine pathway in HIV/AIDS: an immune checkpoint at the crossroads of metabolism and inflammation. AIDS Rev. 2015;17(2):96-106.

35. Dixon DCK, Ratan C, Nair B, Mangalath S, Abraham R, Nath LR. RNA sensors as a mechanism of innate immune evasion among SARS-CoV2, HIV and Nipah viruses. Curr Protein Pept Sci. 2021;22. doi:10.2174/1389203722666210322142725

36. Pelchen-Matthews A, Ryom L, Borges AH, et al. Aging and the evolution of comorbidities among HIV-positive individuals in a European cohort. AIDS. 2018;32(16):2405-2416. doi:10.1097/ QAD.0000000000001967

37. Neri S, Leung J, Besutti G, Santoro A, Fabbri LM, Guaraldi G. Chronic lung disease in HIV patients. AIDS Rev. 2018;20 (3):150-157. doi:10.24875/AIDSRev.18000002

38. Jan AK, Moore JV, Wang RJ, et al. Markers of inflammation and immune activation are associated with lung function in a multi-center cohort of persons with HIV. AIDS. 2021;35(7):1031-1040. doi:10.1097/QAD.0000000000002846
Infection and Drug Resistance

\section{Publish your work in this journal}

Infection and Drug Resistance is an international, peer-reviewed openaccess journal that focuses on the optimal treatment of infection (bacterial, fungal and viral) and the development and institution of preventive strategies to minimize the development and spread of resistance. The journal is specifically concerned with the epidemiology of antibiotic resistance and the mechanisms of resistance development and diffusion in both hospitals and the community. The manuscript management system is completely online and includes a very quick and fair peerreview system, which is all easy to use. Visit http://www.dovepress.com/ testimonials.php to read real quotes from published authors. 